\title{
Diastereoselective synthesis of 6-functionalized 4-aryl-1,3-oxazinan-2-ones and their application in the synthesis of 3-aryl-1,3-aminoalcohols and 6- arylpiperidine-2,4-diones
}

\author{
Sven Mangelinckx, ${ }^{\mathrm{a},}{ }^{\dagger}$ Yahya Nural, ${ }^{\mathrm{b}}$ H. Ali Dondas, ${ }^{\mathrm{b}}$ Bram Denolf, ${ }^{\mathrm{a}}$ Reijo Sillanpää, ${ }^{\mathrm{c}}$ Norbert \\ De Kimpe ${ }^{\mathrm{a}, *}$
}

${ }^{a}$ Department of Organic Chemistry, Faculty of Bioscience Engineering, Ghent University, Coupure Links 653, B-9000, Ghent, Belgium; ${ }^{b}$ Department of Basic Pharmaceutical Sciences, Faculty of Pharmacy, Mersin University, Yenisehir, Mersin, Turkey; ${ }^{c}$ Department of Chemistry, University of Jyväskylä, Fin-40351, Jyväskylä, Finland

\section{Graphical abstract}<smiles>[R]N([R])C([Al])CC=C</smiles>

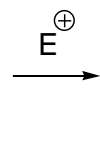<smiles>FCC(CF)CC[Al]</smiles>

$\mathrm{E}=\mathrm{Br}, \mathrm{I}, \mathrm{PhSe}$

$\mathrm{R}=\mathrm{H}, \mathrm{Bn}$ cis/trans $90 / 10-6 / 94$
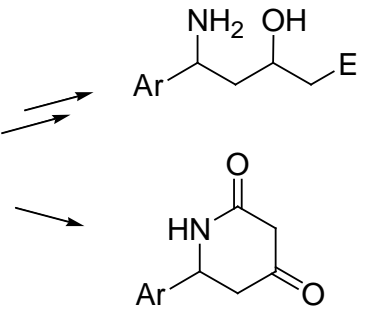

\section{Abstract}

Halocyclocarbamation of benzyl $N$-(1-phenyl-3-butenyl)carbamates afforded 6-functionalized 4-aryl-1,3-oxazinan-2-ones with moderate to excellent diastereoselectivity depending on the $\mathrm{N}$-substituent. Importantly, in contrast to reported cyclocarbamations of homoallylic carbamates which are generally trans-diastereoselective, cyclization of $N$-unsubstituted Cbzprotected homoallylamines led to cis-1,3-oxazinan-2-ones, predominantly. The use of $\mathrm{N}$ benzylated and in situ prepared $N$-silylated derivatives resulted however in trans-selectivity. Transition states are proposed to explain the stereochemical influence of the $N$-substituent on the cyclocarbamations. The functionalized 1,3-oxazinan-2-ones could be further elaborated

* Corresponding author. Tel.: +32 9264 59 51; fax: +32 926462 43; e-mail: norbert.dekimpe@UGent.be 
towards biologically or synthetically important 6-arylpiperidine-2,4-diones and 3-aryl-1,3aminoalcohols.

\section{Introduction}

1,3-Oxazinan-2-ones form an important class of heterocyclic compounds which have been studied for their biological activities, ${ }^{1}$ showing antibacterial, ${ }^{2}$ anti-inflammatory, ${ }^{3}$ anti-HIV, ${ }^{4}$ and antithrombotic activities. ${ }^{5}$ Furthermore, 1,3-oxazinan-2-ones are useful compounds for further synthetic transformations to natural products ${ }^{6}$ or biologically relevant compounds, ${ }^{7}$ and are naturally occurring compounds, e.g. the maytansinoid antitumor compounds. ${ }^{8}$ The closely related 1,3-aminoalcohols, which are suitable precursors of 1,3-oxazinanones, ${ }^{9}$ as well as derived products from the latter heterocycles, ${ }^{10}$ have found wide application as ligands, auxiliaries and phase transfer catalysts in organic chemistry. ${ }^{11}$ One of the two most important methods for the asymmetric synthesis of 1,3-oxazinan-2-ones, next to halocyclocarbamation of chiral homoallylic carbamates, ${ }^{6 a, e-h, 10}$ involves cyclization of chiral 1,3 -aminoalcohols. ${ }^{7 a, 9 a-}$ ${ }^{c, e}$ More specifically, the 3-phenyl-1,3-aminoalcohol moiety is an important C3-unit present in naturally occurring products, such as (-)-cytoxazone (1), ${ }^{12}$ (-)-codonopsinine (2), ${ }^{13}$ the lythraceous alkaloids (-)-lasubine I (3), (-)-lasubine II (4), ${ }^{14}$ and vertine (cryogenine) (5) with ataractic, anti-inflammatory, antispasmodic and antimalarial activity. ${ }^{15}$ Other examples are 1,2-diphenyl substituted diphenylpyraline derivatives cis-6 and trans-6 with good antimycobacterial activity, ${ }^{16}$ the vesicular monoamine transporter type 2 (VMAT2) antagonist dihydrotetrabenazine (DTBZ) as a potent hypoglycemic agent, ${ }^{17}$ and (S)-dapoxetine (7), which is used for treatment of premature ejaculation and is structurally related to fluoxetine (Prozac) (8). ${ }^{18}$ Moreover, 3-phenyl-1,3-aminoalcohols are also useful in the synthesis of druglike azetidines, ${ }^{19}$ and piperidines. ${ }^{20}$ Very recently it was shown that anti-2-alkoxy-3-amino-3arylpropan-1-ols and the corresponding ring closed cis-5-alkoxy-4-aryl-1,3-oxazinanes are promising new types of antimalarial agents. ${ }^{21}$

In the present article, the results of an extensive study on the diastereoselective electrophileinduced cyclocarbamation of different $N$-protected benzyl $N$-(1-phenyl-3-butenyl)carbamates towards the synthesis of cis- or trans-6-(bromomethyl)-, 6-(iodomethyl)- and 6(phenylselenomethyl)-4-phenyl-1,3-oxazinan-2-ones are disclosed. These oxazinan-2-ones represent versatile intermediates for the synthesis of functionalized 3-aryl-1,3-aminoalcohols and 6-arylpiperidine-2,4-diones. 
<smiles>COc1ccc(C2NC(=O)OC2CO)cc1</smiles>

(-)-cytoxazone (1)<smiles>COc1ccc([C@H]2[C@@H](O)C(O)C(C)N2C)cc1</smiles>

(-)-codonopsinine (2)<smiles>COc1ccc([C@@H]2C[C@H](O)C[C@H]3CCCCN23)cc1OC</smiles>

(-)-lasubine I (3)<smiles>COc1ccc([C@@H]2C[C@H](O)C[C@H]3CCCCN23)cc1OC</smiles>

(-)-lasubine II (4)<smiles></smiles>

vertine (cryogenine) (5)<smiles>C/C=C/C=C/CO[C@H]1CCN(c2ccccc2)[C@H](c2ccccc2)C1</smiles>

6<smiles>CNCCC(Oc1ccc(C(F)(F)F)cc1)c1ccccc1</smiles>

(S)-dapoxetine (7)

fluoxetine (Prozac) (8)

\section{Results and discussion}

Despite the fact that electrophile-induced cyclocarbamation of homoallylic carbamates to 4,6disubstituted 1,3-oxazinan-2-ones has been reported regularly in organic synthesis, ${ }^{\text {6a,d-f,h,10b,22 }}$ the method has not been developed in a general sense. Mostly trans-4,6-disubstituted oxazinanones are prepared starting from fully $N$-substituted homoallylic carbamates without the development of a selective preparative method to the cis-diastereomers. ${ }^{6 \mathrm{a}, \mathrm{d}, \mathrm{h}, 10 \mathrm{~b}, 22 \mathrm{a}, \mathrm{b}}$ Moreover, a lack of diastereoselectivity in some cyclocarbamations detracts from synthetic utility. ${ }^{6 \mathrm{~d}, 22 c, \mathrm{~d}}$ Benzyl $N$-(1-phenyl-3-butenyl)carbamates 9 were prepared according to a recently described iodine-catalyzed three-component condensation of benzaldehydes, benzylcarbamate and allyltrimethylsilane, ${ }^{23}$ as suitable starting materials for further diastereoselective electrophile-induced cyclocarbamation to 6-functionalized cis- and trans-4phenyl-1,3-oxazinan-2-ones $\mathbf{1 0 .}$

The benzyl $\mathrm{N}$-(1-phenyl-3-butenyl)carbamates 9 with a free $\mathrm{NH}$ group were directly submitted to cyclocarbamation with bromine, iodine or phenylselenyl bromide in dichloromethane, ${ }^{24}$ furnishing the desired cis- and trans-4-aryl-1,3-oxazinan-2-ones 10a-f in 79-90\% yield after crystallisation with low to good cis-diastereoselectivity (Scheme 1). A decreasing cis/trans ratio from 80:20 to 54:46 was observed as the steric demand of the 6substituent increases from $\mathrm{CH}_{2} \mathrm{Br}$ to $\mathrm{CH}_{2} \mathrm{SePh}$. Column chromatography on silica gel allowed the isolation of the major cis-6-(bromomethyl)- and 6-(iodomethyl)-4-phenyl-1,3-oxazinan-2ones 10a-d as diastereomerically pure compounds in acceptable yields (29-42\%) together 
with the pure trans-isomers 10a-d (7-16\% yield). This result forms one of the few examples in which 4,6-disubstituted 1,3-oxazinan-2-ones resulting from a cis-stereoselective halocyclocarbamation have been isolated in a preparatively useful manner. The structural assignment of the cis- and trans-isomers $\mathbf{1 0}$ is based on analysis of the values of the vicinal coupling constants $\left({ }^{3} J_{\mathrm{H}-\mathrm{H}}\right)$ of $\mathrm{H} 4, \mathrm{H} 5, \mathrm{H} 5$ ' and H6 (Figure 1). The 6-substituent occupies an equatorial position both in the cis- and trans-isomers $\mathbf{1 0}$ as demonstrated by the typical large coupling constants ${ }^{3} J_{\mathrm{H} 6-\mathrm{H} 5}=9.0-11.6 \mathrm{~Hz}$ for the axial protons $\mathrm{H} 5$ and H6. ${ }^{10 \mathrm{~b}, 22 \mathrm{a}}$ For the cisisomers 10, a large coupling constant ${ }^{3} J_{\mathrm{H} 4-\mathrm{H} 5}=11.3-11.6 \mathrm{~Hz}$ for $\mathrm{H} 4$ and $\mathrm{H} 5$ in axial positions is also observed. The structure and the stereochemical arrangement of cis-6(bromomethyl)-4-(4-methoxyphenyl)-1,3-oxazinan-2-one 10b was unambiguously determined by X-ray diffraction analysis (Figure 2).

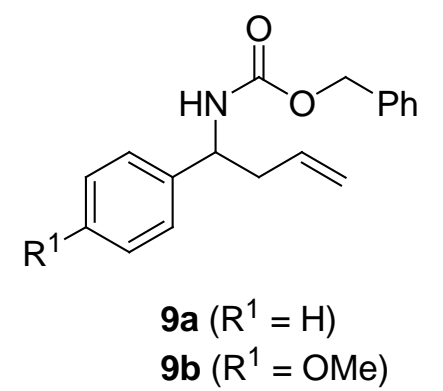

1.22 equiv $E-X$

$\mathrm{CH}_{2} \mathrm{Cl}_{2}, \mathrm{rt}, 16-24 \mathrm{~h}$ $\left(\mathrm{E}-\mathrm{X}=\mathrm{Br}_{2}, \mathrm{I}_{2}, \mathrm{PhSeBr}\right)$<smiles>[R]c1ccc([C@@H]2C[C@H](CF)OC(=O)N2)cc1</smiles>

10a $\left(\mathrm{R}^{1}=\mathrm{H}, \mathrm{E}=\mathrm{Br}, 83 \%\right.$, dr cis/trans 75:25)

10b $\left(\mathrm{R}^{1}=\mathrm{OMe}, \mathrm{E}=\mathrm{Br}, 86 \%\right.$, dr cis/trans $\left.80: 20\right)$

10c $\left(R^{1}=\mathrm{H}, \mathrm{E}=\mathrm{I}, 84 \%\right.$, dr cis/trans $\left.74: 26\right)$

$\operatorname{10d}\left(R^{1}=\right.$ OMe, $E=I, 90 \%$, dr cis/trans 78:22)

10e $\left(R^{1}=\mathrm{H}, \mathrm{E}=\mathrm{SePh}, 79 \%\right.$, dr cis/trans 54:46)

$10 f\left(R^{1}=\mathrm{OMe}, \mathrm{E}=\mathrm{SePh}, 85 \%\right.$, dr cis/trans 58:42)

\section{Scheme 1.}
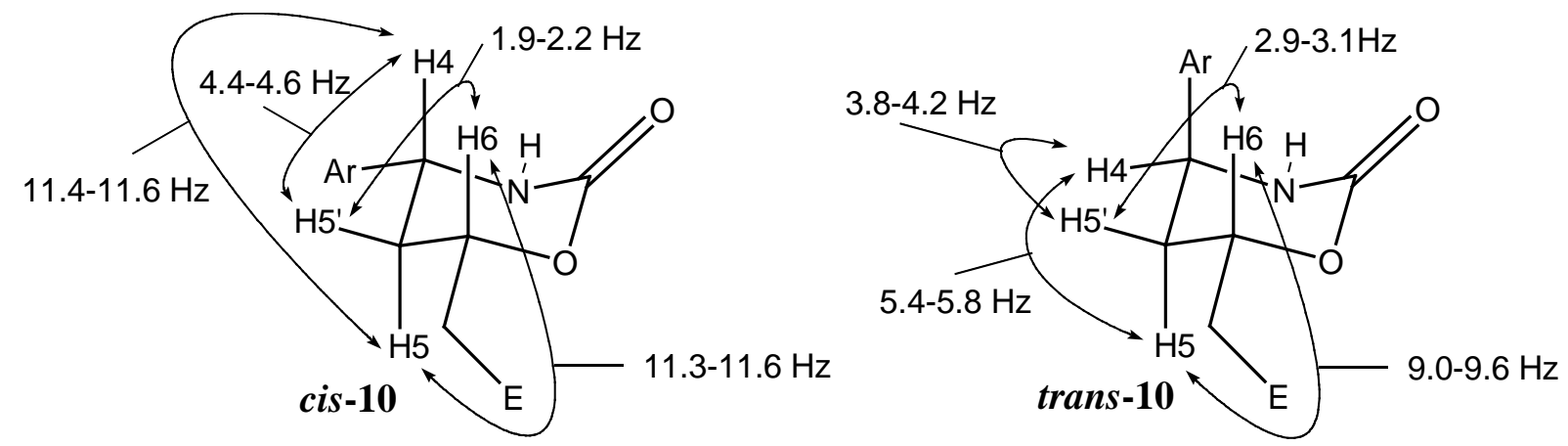

Figure 1. Selected coupling constants for cis- and trans-oxazinanones $\mathbf{1 0}$ 


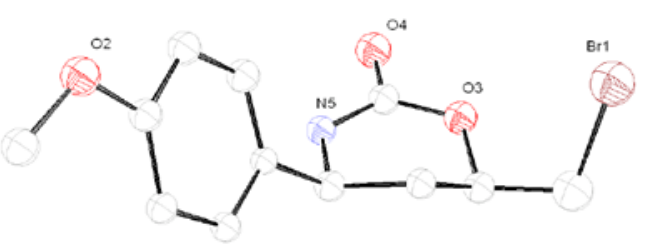

Figure 2. Ortep view of compound cis-10b by X-ray diffraction analysis

Bromocyclocarbamation of homoallylamine 9a with $N$-bromosuccinimide in dichloromethane afforded cis- and trans-6-(bromomethyl)-4-phenyl-1,3-oxazinan-2-ones 10a in a good cis/trans ratio of 9:1 (Scheme 2). However, the reaction time increased to 48 hours and the yield dropped to $74 \%$ after extractive work up with $2 \mathrm{M}$ aq. $\mathrm{NaOH}$ and crystallization from dichloromethane and hexane. The latter steps were necessary to convert the unstable intermediate 2-benzyloxy-6-(bromomethyl)-4-phenyl-5,6-dihydro-4H-[1,3]oxazines into the 1,3-oxazinan-2-ones 10a and to remove the formed succinimide and benzyl alcohol. An attempted chlorocyclocarbamation of homoallylamine $\mathbf{9 b}$ with $N$-chlorosuccinimide failed, as no reaction occurred upon stirring carbamate $\mathbf{9 b}$ with 1.22 equiv NCS at room temperature in dichloromethane for 10 days.<smiles>C=CCC(NC(=O)OCc1ccccc1)c1ccccc1</smiles>

$9 a$
1.22 equiv NBS

$\mathrm{CH}_{2} \mathrm{Cl}_{2}, \mathrm{rt}, 48 \mathrm{~h}$

10a (74\%, dr cis/trans 9:1)

Scheme 2.

The diastereoselectivity of the cyclocarbamation of the benzyl $N$-(1-phenyl-3butenyl)carbamates $\mathbf{9}$ was nicely reversed after in situ protection of the homoallylamines $\mathbf{9}$ with a tert-butyldimethylsilyl (TBDMS) group. ${ }^{6 a}$ Treatment of the homoallylamines 9 with TBDMS triflate in the presence of 2,6-lutidine in dichloromethane at $0{ }^{\circ} \mathrm{C}$ for 2 hours, followed by reaction with excess of bromine, iodine or phenylselenyl bromide at $0{ }^{\circ} \mathrm{C}$ for 16 - 
24 hours afforded the cis- and trans-4-aryl-1,3-oxazinan-2-ones 10a-f in 71-76\% yield after crystallisation with trans-diastereoselectivity (Scheme 3). In the bromocyclocarbamation of benzyl $N$-(1-(4-methoxyphenyl)-3-butenyl)carbamates $\mathbf{9 b}$, the typical excess of 2.44 equiv of electrophile was lowered to 2.05 equiv of $\mathrm{Br}_{2}$ to avoid bromination in the ortho-position of the aromatic methoxy substituent. The highest trans-diastereoselectivity, up to trans/cis ratio 9:1, was obtained in the iodocyclocarbamation to 6-(iodomethyl)-4-phenyl-1,3-oxazinan-2ones 10c,d. A slight trans-selectivity was observed for the selenides 10e,f.<smiles>[R]c1ccc(C(CC=C)NC(=O)OCc2ccccc2)cc1</smiles>

1) 3 equiv TBDMSOTf 4 equiv 2,6 -lutidine $\mathrm{CH}_{2} \mathrm{Cl}_{2}, 0^{\circ} \mathrm{C}, 2 \mathrm{~h}$

2) 2.05-2.44 equiv $\mathrm{E}-\mathrm{X}$ $\mathrm{CH}_{2} \mathrm{Cl}_{2}, 0^{\circ} \mathrm{C}, 16-24 \mathrm{~h}$ $\left(\mathrm{E}-\mathrm{X}=\mathrm{Br}_{2}, \mathrm{I}_{2}, \mathrm{PhSeBr}\right)$<smiles>[R]c1ccc([C@@H]2C[C@H](CF)OC(=O)N2)cc1</smiles>

10a $\left(\mathrm{R}^{1}=\mathrm{H}, \mathrm{E}=\mathrm{Br}, 76 \%\right.$, dr cis/trans 24:76)

10b $\left(\mathrm{R}^{1}=\mathrm{OMe}, \mathrm{E}=\mathrm{Br}, 73 \%\right.$, dr cis/trans $\left.30: 70\right)$

10c $\left(R^{1}=H, E=I, 71 \%\right.$, dr cis/trans 10:90)

10d $\left(R^{1}=\right.$ OMe, $E=I, 75 \%$, dr cis/trans 15:85)

10e $\left(R^{1}=\mathrm{H}, \mathrm{E}=\mathrm{SePh}, 73 \%\right.$, dr cis/trans 46:54)

$10 f\left(R^{1}=\right.$ OMe, $\mathrm{E}=\mathrm{SePh}, 71 \%$, dr cis/trans 44:56)

Scheme 3.

Similarly, the $N$-benzyl-substituted homoallylamines 11, prepared in 65-70\% yield by protection of homoallylamines 9 with benzyl iodide after deprotonation with $\mathrm{NaH}$ in $\mathrm{DMF},{ }^{25}$ underwent cyclocarbamation with zero to excellent trans-diastereoselectivity (trans/cis ratio from 50:50 to 94:6). ${ }^{6 a}$ The trans- and cis-4-aryl-3-benzyl-1,3-oxazinan-2-ones 12a-f were obtained in 78-86\% yield after column chromatography (Scheme 4).<smiles>[R]c1ccc(C(CC=C)N([R])C(=O)OCc2ccccc2)cc1</smiles>

1.22 equiv $E-X$

$\mathrm{CH}_{2} \mathrm{Cl}_{2}, \mathrm{rt}, 18-40 \mathrm{~h}$ $\left(\mathrm{E}-\mathrm{X}=\mathrm{Br}_{2}, \mathrm{I}_{2}, \mathrm{PhSeBr}\right)$<smiles>[R]c1ccc([C@@H]2C[C@H](CF)OC(=O)N2Cc2ccccc2)cc1</smiles>

12a $\left(R^{1}=\mathrm{H}, \mathrm{E}=\mathrm{Br}, 86 \%\right.$, dr cis/trans 11:89)

12b $\left(\mathrm{R}^{1}=\mathrm{OMe}, \mathrm{E}=\mathrm{Br}, 85 \%\right.$, dr cis/trans $\left.15: 85\right)$

12c $\left(R^{1}=H, E=I, 85 \%\right.$, dr cis/trans 6:94)

12d $\left(R^{1}=\right.$ OMe, $E=I, 86 \%$, dr cis/trans 9:91)

12e $\left(R^{1}=\mathrm{H}, \mathrm{E}=\mathrm{SePh}, 78 \%\right.$, dr cis/trans 33:67)

$12 f\left(R^{1}=\right.$ OMe, $E=$ SePh, 82\%, dr cis/trans 50:50)
$11 \mathrm{a}\left(\mathrm{R}=\mathrm{Bn}, \mathrm{R}^{1}=\mathrm{H}, 70 \%\right)$

11b $\left(R=B n, R^{1}=\right.$ OMe, $\left.65 \%\right)$

\section{Scheme 4.}

9a $\left(\mathrm{R}=\mathrm{R}^{1}=\mathrm{H}\right)$

9b $\left(R=H, R^{1}=O M e\right)$

2) 17 equiv $\mathrm{Bnl}$

DMF, rt, $10 \mathrm{~min}$ 
The cis-stereoselectivity of the cyclocarbamation reactions of the N-monosubstituted homoallylamines $\mathbf{9}$ can be explained on the basis of the conformational arrangement in the proposed transition state of the reaction (Figure 3). ${ }^{6 \mathrm{~d}}$ The major cis-1,3-oxazinan-2-ones 10 are probably formed through half-chair type transition state $\mathbf{1 3}$ in which both the alkene moiety, undergoing the anti-attack of the carbonyl oxygen, as well as the 4-aryl substituent adopt pseudoequatorial positions with only minor 1,2-strain between the $\mathrm{N}$-H substituent and the neighbouring 4-aryl substituent. The introduction of a second substituent on nitrogen, either via in situ $N$-silylation of carbamates 9 with TBDMSOTf or via $N$-benzylation to carbamates 11, forces the 4-aryl substituent to adopt a pseudoaxial position. In this way the 1,2-strain with the neighbouring $N$-TBDMS or $N$-benzyl substituent is minimized in transition state 14 resulting in a small 1,3-diaxial interaction, and the formation of the trans-1,3oxazinanones $\mathbf{1 0}$ and $\mathbf{1 2}$ is preferred.

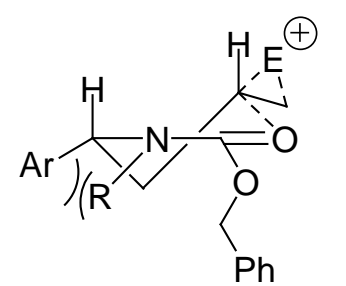

13

favored if $\mathrm{R}=\mathrm{H}$

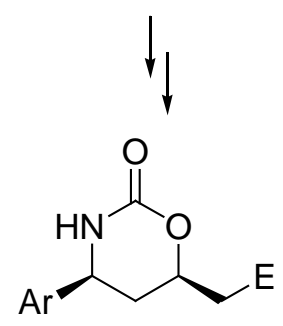

cis-10

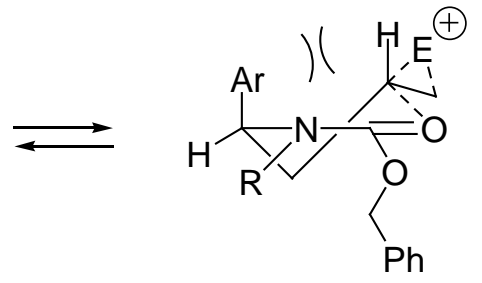

14

favored if $\mathrm{R}=$ TBDMS or $\mathrm{Bn}$

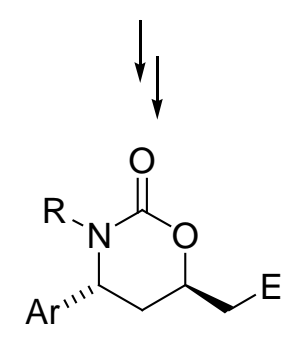

trans-10 or trans-12

Figure 3. Possible transition states in the formation of the cis- and trans-diastereomers $\mathbf{1 0}$ and $\mathbf{1 2}$

Having the functionalized 1,3-oxazinan-2-ones $\mathbf{1 0}$ in hand, their synthetic potential was studied under different conditions. A typical attempt to hydrolyze the 6-(iodomethyl)-1,3oxazinan-2-ones 10c,d directly to the corresponding 1,3 -aminoalcohols, ${ }^{10 a}$ afforded an unexpected but interesting new synthesis of 6-arylpiperidine-2,4-diones. Treatment of 4-aryl6-(iodomethyl)-1,3-oxazinan-2-ones $\mathbf{1 0}$, $\mathbf{d}$ with an aqueous solution of sodium hydroxide under reflux in ethanol afforded the 6-arylpiperidine-2,4-diones 15 in 82-86\% yield after 
recrystallisation (Scheme 5). 6-Arylpiperidine-2,4-diones have been prepared as analogues of VMAT2 antagonists, ${ }^{26}$ and represent key intermediates for further synthetic transformations to 6-aryl-4-hydroxypiperidin-2-ones, 27 4-hydroxypipecolic acids, $28 \quad(R)-(+)-2-$ phenylpiperidine, ${ }^{29}$ and biomimetic NADH models. ${ }^{30}$ Relatively few methods for the synthesis of 6-arylpiperidine-2,4-diones have been reported in the literature. An important method is the coupling of $\beta$-aryl- $\beta$-amino esters with alkyl malonates, followed by Dieckmann condensation, hydrolysis and decarboxylation. ${ }^{27}$ Alternatively, $\beta$-aryl- $\beta$-amino acid derivatives can be reacted with metal enolates of alkyl acetates or with Meldrum's acid in the presence of pyridine to afford the corresponding $\delta$-amino- $\beta$-keto esters which are subsequently cyclized under basic conditions to give 6-arylpiperidine-2,4-diones. ${ }^{29,31}$ Analogously, a number of 6-arylpiperidine-2,4-diones were obtained by reaction of chiral $\mathrm{N}$ sulfinyl imines with lithium or TMS dienolates of 2,2,6-trimethyl-1,3-dioxin-4-one. ${ }^{32}$ An early efficient preparation of 1-tert-butyl-6-phenylpiperidine-2,4-dione involved the addition of $N$-benzylidene-tert-butylamine to diketene. ${ }^{33} 1$-Methyl-6-phenylpiperidine-2,4-dione was synthesized in moderate yield through 1,3-dipolar cycloaddition of the appropriate nitrone with methyl 2-chlorobut-3-enoate and subsequent hydrogenolysis of the intermediate isoxazolidine. ${ }^{34}$ 6-Arylpiperidine-2,4-diones are also accessible by acid hydrolysis of the cycloadducts obtained through aza-Diels-Alder reaction of 1,3-dimethoxy-1(trimethylsiloxy)butadiene (Brassard's diene) with aromatic aldimines. ${ }^{35}$ The formation of piperidine-2,4-diones 15 from 6-(iodomethyl)-1,3-oxazinan-2-ones $\mathbf{1 0}$ is believed to proceed via initial base-induced elimination of hydrogen iodide to give 6-methylene-1,3-oxazinan-2ones 16 followed by basic hydrolysis of the oxazinanone and intramolecular $C$ carbamoylation of the formed enolate. The alkoxide-catalyzed rearrangement of isomeric 2alkylidene-1,3-oxazinan-6-ones to piperidine-2,4-diones has been described more than 40 years ago. ${ }^{36}$ On the other hand, to the best of our knowledge, the rearrangement of 6alkylidene-1,3-oxazinan-2-ones to piperidine-2,4-diones has never been reported.

Alternatively, the iodo substituent in 6-(iodomethyl)-1,3-oxazinan-2-ones 10c,d could be successfully substituted upon heating with sodium azide in DMSO which efficiently afforded the corresponding 6-(azidomethyl)-1,3-oxazinan-2-ones 17 (Scheme 5). The absence of a tethered leaving group in the C-6 substituent of 6-(azidomethyl)-1,3-oxazinan-2-ones 17, inhibiting base-induced elimination to 6-methylene-1,3-oxazinan-2-ones 16, allowed the solvolysis to 4-amino-4-aryl-1-azidobutan-2-ols 18 (81-90\% yield) upon heating with sodium methoxide in methanol. Upon considering the aryl group as a carboxyl synthon, ${ }^{37}$ the azido aminoalcohols $\mathbf{1 8}$ are of potential interest for further synthetic elaboration as they incorporate 
the scaffold of the naturally occurring nonproteinogenic amino acids 4-hydroxyornithine and 4-hydroxyarginine, ${ }^{38}$ which are also constituents of the antibiotic cyclopeptide natural products biphenomycins, ${ }^{39} \mathrm{~K}-582$ type antibiotics, ${ }^{40}$ and $\beta$-lactam antibiotic clavalanine. ${ }^{41}$<smiles>[R20]c1ccc([C@@H]2C[C@H](CI)OC(=O)N2)cc1</smiles>

10c $\left(\mathrm{R}^{1}=\mathrm{H}\right.$, dr cis/trans 74:26)

10d $\left(\mathrm{R}^{1}=\mathrm{OMe}\right.$, dr cis/trans 78:22)<smiles>[R]c1ccc([C@H]2CC(=C)OC(=O)C2)cc1</smiles>

$$
\begin{aligned}
& 2 \text { equiv } \mathrm{NaN}_{3} \\
& \text { DMSO, } 80^{\circ} \mathrm{C}, 14 \mathrm{~h}
\end{aligned}
$$<smiles>[R]c1ccc([C@@H]2C[C@H](CN)OC(=O)N2)cc1</smiles>

17a $\left(R^{1}=H, 81 \%\right.$, dr cis/trans $\left.69: 31\right)$

17b $\left(\mathrm{R}^{1}=\mathrm{OMe}, 85 \%\right.$, dr cis/trans 82:18)
4 equiv $2 \mathrm{M} \mathrm{NaOMe} / \mathrm{MeOH}$<smiles>[R1]c1ccc([C@@H](N)C[C@@H](O)CN)cc1</smiles>

18a $\left(R^{1}=\mathrm{H}, 90 \%\right.$, dr syn/anti 72:28)

18b $\left(\mathrm{R}^{1}=\mathrm{OMe}, 81 \%\right.$, dr synlanti $\left.>90: 10\right)$

\section{Scheme 5.}

Similarly, the 6-(phenylselanylmethyl)-1,3-oxazinan-2-ones 10e,f were easily solvolyzed to the corresponding 4-amino-4-aryl-1-(phenylselanyl)butan-2-ols 19 (80-84\% yield) (Scheme 6). The latter 1,3-aminoalcohols 19 are of synthetic interest in view of the versatile chemistry of organoselenium compounds. ${ }^{42}$<smiles>[R]c1ccc([C@@H]2C[C@H](C[SeH]c3ccccc3)OC(=O)N2)cc1</smiles>

10e $\left(\mathrm{R}^{1}=\mathrm{H}\right.$, dr cis/trans $\left.46: 54\right)$ $10 f\left(R^{1}=\mathrm{MeO}\right.$, dr cis/trans 44:56)

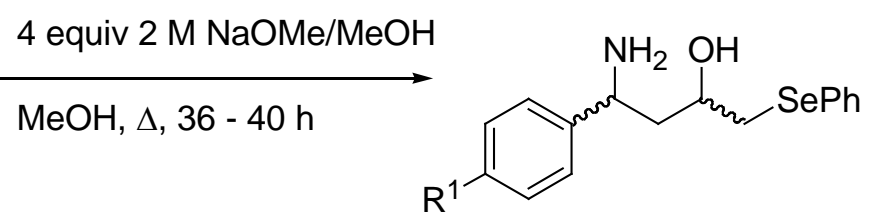

19a $\left(\mathrm{R}^{1}=\mathrm{H}, 84 \%\right.$, dr syn/anti 2:3) 19b $\left(\mathrm{R}^{1}=\mathrm{MeO}, 80 \%\right.$, dr syn/anti 49:51)

\section{Scheme 6.}

\section{Conclusion}


In conclusion, an efficient synthesis of 6-functionalized 4-aryl-1,3-oxazinan-2-ones has been achieved based on electrophile-induced cyclocarbamation of benzyl $N$-(1-phenyl-3butenyl)carbamates. Most importantly, stereochemically pure cis-6-(bromomethyl)- and 6(iodomethyl)-4-phenyl-1,3-oxazinan-2-ones became accessible via cyclization of $\mathrm{N}$ unsubstituted Cbz-protected homoallylamines. On the other hand, the use of $N$-benzylated and in situ prepared $N$-silylated derivatives resulted in cyclocarbamation with trans-selectivity. Furthermore, the functionalized 1,3-oxazinan-2-ones could be elaborated towards biologically or synthetically important 6-arylpiperidine-2,4-diones and 3-aryl-1,3-aminoalcohols.

\section{Experimental}

\subsection{General}

${ }^{1} \mathrm{H}$ NMR spectra were recorded at $300 \mathrm{MHz}$ with $\mathrm{CDCl}_{3}$ as solvent and tetramethylsilane as internal standard. ${ }^{13} \mathrm{C}$ NMR spectra were recorded at $75 \mathrm{MHz}$ with $\mathrm{CDCl}_{3}$ as solvent. Dichloromethane was distilled over $\mathrm{CaH}_{2}$, DMF was distilled and kept over molecular sieves, methanol was dried by reaction with magnesium and distilled, while other solvents were used as received from the supplier.

\subsection{Synthetic procedures}

4.2.1. Synthesis of benzyl $N$-(1-phenyl-3-butenyl)carbamates 9. Benzyl $N$-(1-phenyl-3butenyl)carbamates $\mathbf{9}$ were prepared according to a literature procedure. ${ }^{23}$

4.2.2. General procedure for the preparation of 4-aryl-1,3-oxazinan-2-ones 10. A solution of electrophile (1.22 mmol) $\left(\mathrm{Br}_{2}, \mathrm{I}_{2}, \mathrm{PhSeBr}\right)$ in freshly distilled dry dichloromethane $(10 \mathrm{~mL})$ was added dropwise in a period of 10 minutes to a stirred solution of benzyl $N$-(1-phenyl-3butenyl)carbamate $9(1.0 \mathrm{mmol})$ in dichloromethane $(20 \mathrm{~mL})$ and the mixture was stirred at room temperature under nitrogen atmosphere for $16-24$ hours. The reaction was quenched with $2 \mathrm{~N}$ aq. $\mathrm{Na}_{2} \mathrm{SO}_{3}$ (for reaction with $\mathrm{Br}_{2}$ ) or $2 \mathrm{~N}$ aq. $\mathrm{Na}_{2} \mathrm{~S}_{2} \mathrm{O}_{3}$ (for reaction with $\mathrm{I}_{2}$ ) or brine (for reaction with $\mathrm{PhSeBr}$ ). The mixture was extracted with dichloromethane, dried $\left(\mathrm{MgSO}_{4}\right)$, filtered, evaporated under reduced pressure and the residue was crystallized from diethyl ether/hexane to afford 4-aryl-1,3-oxazinan-2-ones $\mathbf{1 0}$ as a mixture of cis- and trans-isomers. 
4.2.3. cis-6-(Bromomethyl)-4-phenyl-1,3-oxazinan-2-one (10a). Isolated by column chromatography (diethyl ether). Amorphous white solid; mp: $183.0-185.0{ }^{\circ} \mathrm{C}$; yield $42 \% .{ }^{1} \mathrm{H}$ NMR (300 MHz, $\mathrm{CDCl}_{3}$ ): $\delta=1.86$ (dt, 1H, $J=13.76 \mathrm{~Hz}, 11.56 \mathrm{~Hz}, 5-\mathrm{CH}(\mathrm{H})$ ), 2.47 (dddd, $1 \mathrm{H}, J=13.76 \mathrm{~Hz}, 4.40 \mathrm{~Hz}, 2.20 \mathrm{~Hz}, 1.65 \mathrm{~Hz}, 5-\mathrm{CH}(H)$ ), 3.46 (dd, $1 \mathrm{H}, J=10.73 \mathrm{~Hz}, 6.88 \mathrm{~Hz}$, $\mathrm{CH}(\mathrm{H}) \mathrm{Br}$ ), 3.60 (dd, $1 \mathrm{H}, J=10.73 \mathrm{~Hz}, 4.40 \mathrm{~Hz}, \mathrm{CH}(H) \mathrm{Br}$ ), 4.57-4.65 (m, 1H, CHO), 4.63 (dd, $1 \mathrm{H}, J=11.70 \mathrm{~Hz}, 4.5 \mathrm{~Hz}, \mathrm{CHN}$ ), 5.32 (br s, $1 \mathrm{H}, \mathrm{NH}$ ), 7.31-7.45 (m, 5H, $\mathrm{C}_{6} \mathrm{H}_{5}$ ). ${ }^{13} \mathrm{C}$ NMR (75 MHz, $\mathrm{CDCl}_{3}$ ): $\delta=32.6,35.3,55.2,75.8,126.2,129.0,129.3,140.2$, 153.1. IR

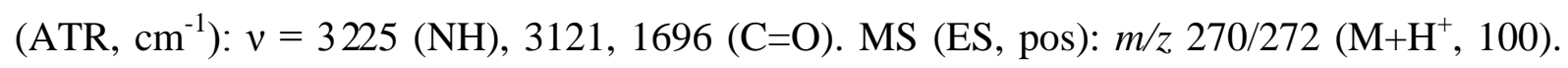
Anal. Calcd. for $\mathrm{C}_{11} \mathrm{H}_{12} \mathrm{BrNO}_{2}$ : C, 48.91; H, 4.48; N, 5.19. Found: C, 48.87; H, 4.09; N, 5.06.

4.2.4. trans-6-(Bromomethyl)-4-phenyl-1,3-oxazinan-2-one (10a). Isolated by column chromatography (diethyl ether). Amorphous white solid; mp: 157.1-159.1 ${ }^{\circ} \mathrm{C}$; yield $14 \% .{ }^{1} \mathrm{H}$ NMR (300 MHz, $\mathrm{CDCl}_{3}$ ): $\delta=2.17$ (dddd, $1 \mathrm{H}, J=13.9 \mathrm{~Hz}, 3.9 \mathrm{~Hz}, 3.0 \mathrm{~Hz}, 1.1 \mathrm{~Hz}, 5-\mathrm{CH}(\mathrm{H})$ ), 2.40 (ddd, $1 \mathrm{H}, J=14.0 \mathrm{~Hz}, 9.6 \mathrm{~Hz}, 5.8 \mathrm{~Hz}, 5-\mathrm{CH}(H)$ ), 3.48 (dd, $1 \mathrm{H}, J=10.8 \mathrm{~Hz}, 6.7 \mathrm{~Hz}$, $\mathrm{CH}(\mathrm{H}) \mathrm{Br}$ ), 3.53 (dd, $1 \mathrm{H}, J=10.8 \mathrm{~Hz}, 4.95 \mathrm{~Hz}, \mathrm{CH}(H) \mathrm{Br}$ ), 4.42 (dddd, $1 \mathrm{H}, J=9.5 \mathrm{~Hz}, 6.8 \mathrm{~Hz}$, $4.9 \mathrm{~Hz}, 2.8 \mathrm{~Hz}, \mathrm{CHO}$ ), 4.79 (dt, 1H, $J=5.5 \mathrm{~Hz}, 3.6 \mathrm{~Hz}, \mathrm{CHN}$ ), 5.48 (br s, 1H, NH), 7.29-7.45 (m, 5H, $\left.\mathrm{C}_{6} \mathrm{H}_{5}\right) .{ }^{13} \mathrm{C}$ NMR $\left(75 \mathrm{MHz}, \mathrm{CDCl}_{3}\right): \delta=32.3,32.8,52.3,72.4,125.9,128.4,129.2$, 141.2, 153.2. IR (ATR, $\left.\mathrm{cm}^{-1}\right): v=3243(\mathrm{NH}), 3124,1690$ (C=O). MS (ES, pos): $\mathrm{m} / \mathrm{z}$ 270/272 $\left(\mathrm{M}+\mathrm{H}^{+}, 100\right)$. Anal. Calcd. for $\mathrm{C}_{11} \mathrm{H}_{12} \mathrm{BrNO}_{2}$ : C, 48.91; H, 4.48; N, 5.19. Found: C, 48.75; H, 4.29; N, 5.39 .

4.2.5. cis-6-(Bromomethyl)-4-(4-methoxyphenyl)-1,3-oxazinan-2-one (10b). Isolated by column chromatography (diethyl ether). Colorless crystals; mp: 138.2-140.2 ${ }^{\circ} \mathrm{C}$; yield 36\%. ${ }^{1} \mathrm{H}$ NMR (300 MHz, $\mathrm{CDCl}_{3}$ ): $\delta=1.83$ (dt, $1 \mathrm{H}, J=13.76 \mathrm{~Hz}, 11.56 \mathrm{~Hz}, 5-\mathrm{CH}(\mathrm{H})$ ), 2.41 (dddd, $1 \mathrm{H}, J=13.7 \mathrm{~Hz}, 4.2 \mathrm{~Hz}, 2.0 \mathrm{~Hz}, 2.0 \mathrm{~Hz}, 5-\mathrm{CH}(H)), 3.46$ (dd, $1 \mathrm{H}, J=10.73 \mathrm{~Hz}, 6.88 \mathrm{~Hz}$, $\mathrm{CH}(\mathrm{H}) \mathrm{Br}$ ), 3.59 (dd, $1 \mathrm{H}, J=10.73 \mathrm{~Hz}, 4.40 \mathrm{~Hz}, \mathrm{CH}(\mathrm{H}) \mathrm{Br}$ ), 3.82 (s, 3H, $\mathrm{CH}_{3} \mathrm{O}$ ), 4.54-4.62 (m, 1H, CHO), 4.57 (dd, 1H, J = 11.56 Hz, 4.68 Hz, CHN), 5.46 (br s, 1H, NH), 6.89-6.94 (m, 2H, Ar-H), 7.23-7.28 (m, 2H, Ar-H). ${ }^{13} \mathrm{C}$ NMR (75 MHz, $\left.\mathrm{CDCl}_{3}\right): \delta=32.6,35.4,54.7,55.5,75.8$, 114.6, 127.5, 132.1, 153.0, 160.0. IR (ATR, $\left.\mathrm{cm}^{-1}\right): v=3222(\mathrm{NH}), 3116,1694(\mathrm{C}=\mathrm{O})$. MS (ES, $\mathrm{M}+\mathrm{H}^{+}$, pos): $\mathrm{m} / \mathrm{z} 300 / 302\left(\mathrm{M}+\mathrm{H}^{+}, 100\right)$. Anal. Calcd. for $\mathrm{C}_{12} \mathrm{H}_{14} \mathrm{BrNO}_{3}: \mathrm{C}, 48.02 ; \mathrm{H}$, 4.70; N, 4.67. Found: C, 48.34; H, 4.36; N, 4.55. 
4.2.6. trans-6-(Bromomethyl)-4-(4-methoxyphenyl)-1,3-oxazinan-2-one (10b). Isolated by column chromatography (diethyl ether). White crystals; mp: $146.0-148.0{ }^{\circ} \mathrm{C}$; yield $11 \% .{ }^{1} \mathrm{H}$ NMR (300 MHz, $\mathrm{CDCl}_{3}$ ): $\delta=2.13$ (dddd, $1 \mathrm{H}, J=14.0 \mathrm{~Hz}, 4.1 \mathrm{~Hz}, 3.0 \mathrm{~Hz}, 1.0 \mathrm{~Hz}, 5-\mathrm{CH}(\mathrm{H})$ ), 2.36 (ddd, $1 \mathrm{H}, J=13.9 \mathrm{~Hz}, 9.2 \mathrm{~Hz}, 5.6 \mathrm{~Hz}, 5-\mathrm{CH}(H)$ ), 3.48 (dd, $1 \mathrm{H}, J=10.7 \mathrm{~Hz}, 6.9 \mathrm{~Hz}$, $\mathrm{CH}(\mathrm{H}) \mathrm{Br}$ ), 3.53 (dd, $1 \mathrm{H}, J=10.7 \mathrm{~Hz}, 5.0 \mathrm{~Hz}, \mathrm{CH}(\mathrm{H}) \mathrm{Br}$ ), 3.82 (s, 3H, $\mathrm{CH}_{3} \mathrm{O}$ ), 4.43 (dddd, $1 \mathrm{H}$, $J=9.3 \mathrm{~Hz}, 6.8 \mathrm{~Hz}, 4.8 \mathrm{~Hz}, 2.7 \mathrm{~Hz}, \mathrm{CHO}$ ), 4.74 (m, 1H, CHN), 5.41 (br s, 1H, NH), 6.90-6.95 (m, 2H, Ar-H), 7.21-7.24 (m, 2H, Ar-H). $\left.{ }^{13} \mathrm{C} \mathrm{NMR} \mathrm{(75} \mathrm{MHz,} \mathrm{CDCl}_{3}\right): \delta=32.2,32.9,51.8$, 55.5, 72.5, 114.5, 127.0, 133.1, 153.1, 159.6. IR (ATR, $\left.\mathrm{cm}^{-1}\right): v=3242(\mathrm{NH}), 3125,1674$ $(\mathrm{C}=\mathrm{O})$. MS (ES, $\mathrm{M}+\mathrm{H}^{+}$, pos): $\mathrm{m} / \mathrm{z} 300 / 302\left(\mathrm{M}+\mathrm{H}^{+}, 100\right)$. Anal. Calcd. for $\mathrm{C}_{12} \mathrm{H}_{14} \mathrm{BrNO}_{3}$ : $\mathrm{C}$, 48.02; H, 4.70; N, 4.67. Found: C, 48.40; H, 4.58; N, 4.58.

4.2.7. cis-6-(Iodomethyl)-4-phenyl-1,3-oxazinan-2-one (10c). Isolated by column chromatography (diethyl ether). Colorless crystals; mp: $181.5-183.5{ }^{\circ} \mathrm{C}$; yield $31 \% .{ }^{1} \mathrm{H}$ NMR (300 MHz, $\left.\mathrm{CDCl}_{3}\right): \delta=1.80(\mathrm{dt}, 1 \mathrm{H}, J=13.76 \mathrm{~Hz}, 11.56 \mathrm{~Hz}, 5-\mathrm{CH}(\mathrm{H})$ ), 2.52 (ddt, $1 \mathrm{H}, J=$ 13.76 Hz, $4.40 \mathrm{~Hz}, 1.93 \mathrm{~Hz}, 5-\mathrm{CH}(H)$ ), 3.29 (dd, 1H, $J=10.46 \mathrm{~Hz}, 7.43 \mathrm{~Hz}, \mathrm{CH}(\mathrm{H}) \mathrm{I}), 3.43$ (dd, 1H, $J=10.46 \mathrm{~Hz}, 4.40 \mathrm{~Hz}, \mathrm{CH}(H) \mathrm{I}$ ), 4.44 (dddd, 1H, $J=11.56 \mathrm{~Hz}, 7.02 \mathrm{~Hz}, 4.54 \mathrm{~Hz}, 2.3$ Hz, CHO), 4.63 (dd, 1H, $J=11.56$ Hz, 4.68 Hz, CHN), 5.25 (br s, 1H, NH), 7.31-7.45 (m, 5H, $\left.\mathrm{C}_{6} \mathrm{H}_{5}\right) .{ }^{13} \mathrm{C}$ NMR $\left(75 \mathrm{MHz}, \mathrm{CDCl}_{3}\right): \delta=5.5,36.9,55.2,76.1,126.2,129.0,129.3,140.2$, 153.1. IR (ATR, $\left.\mathrm{cm}^{-1}\right): v=3223(\mathrm{NH}), 3121,1696(\mathrm{C}=\mathrm{O})$. MS (ES, $\mathrm{M}+\mathrm{H}^{+}$, pos): $\mathrm{m} / \mathrm{z} 318$ $\left(\mathrm{M}+\mathrm{H}^{+}, 100\right)$. Anal. Calcd. for $\mathrm{C}_{11} \mathrm{H}_{12} \mathrm{INO}_{2}$ : C, 41.66; H, 3.81; N, 4.42. Found: C, 41.52; H, 3.45; N, 4.29 .

4.2.8. trans-6-(Iodomethyl)-4-phenyl-1,3-oxazinan-2-one (10c). Isolated by column chromatography (diethyl ether). Amorphous white solid; mp: $158.0-160.0{ }^{\circ} \mathrm{C}$; yield $16 \% .{ }^{1} \mathrm{H}$ NMR (300 MHz, $\mathrm{CDCl}_{3}$ ): $\delta=2.20$ (dddd, $1 \mathrm{H}, J=13.9 \mathrm{~Hz}, 4.0 \mathrm{~Hz}, 3.0 \mathrm{~Hz}, 1.0 \mathrm{~Hz}, 5-\mathrm{CH}(\mathrm{H})$ ), 2.37 (ddd, 1H, $J=13.9 \mathrm{~Hz}, 9.2 \mathrm{~Hz}, 5.8 \mathrm{~Hz}, 5-\mathrm{CH}(H)$ ), 3.31 (dd, $1 \mathrm{H}, J=10.73 \mathrm{~Hz}, 6.88 \mathrm{~Hz}$, $\mathrm{CH}(\mathrm{H}) \mathrm{I}$ ), 3.36 (dd, $1 \mathrm{H}, J=10.73 \mathrm{~Hz}, 4.95 \mathrm{~Hz}, \mathrm{CH}(H) \mathrm{I}$ ), 4.24 (dddd, $1 \mathrm{H}, J=9.2 \mathrm{~Hz}, 7.1 \mathrm{~Hz}$, $5.0 \mathrm{~Hz}, 3.0 \mathrm{~Hz}, \mathrm{CHO}$ ), 4.76 (dt, 1H, $J=5.8 \mathrm{~Hz}, 3.6 \mathrm{~Hz}, \mathrm{CHN}$ ), 5.40 (br s, 1H, NH), 7.30-7.45 (m, 5H, $\left.\mathrm{C}_{6} \mathrm{H}_{5}\right) .{ }^{13} \mathrm{C}$ NMR (75 $\left.\mathrm{MHz} \mathrm{CDCl}_{3}\right): \delta=5.3,34.4,52.3,72.7,125.8,128.4,129.2$, 141.2, 153.2. IR (ATR, $\left.\mathrm{cm}^{-1}\right): v=3322(\mathrm{NH}), 1662(\mathrm{C}=\mathrm{O})$. MS (ES, $\mathrm{M}^{+} \mathrm{H}^{+}$, pos): $\mathrm{m} / \mathrm{z} 318$ $\left(\mathrm{M}+\mathrm{H}^{+}, 100\right)$. Anal. Calcd. for $\mathrm{C}_{11} \mathrm{H}_{12} \mathrm{INO}_{2}$ : C, 41.66; H, 3.81; N, 4.42. Found: C, 41.99; H, 3.50; N, 4.33 . 
4.2.9. cis-6-(Iodomethyl)-4-(4-methoxyphenyl)-1,3-oxazinan-2-one (10d). Isolated by column chromatography (diethyl ether). White amorphous solid; mp: 159.4-161.4 ${ }^{\circ} \mathrm{C}$; yield 29\%. ${ }^{1} \mathrm{H}$ NMR (300 MHz, $\mathrm{CDCl}_{3}$ ): $\delta=1.78(\mathrm{dt}, 1 \mathrm{H}, J=14.03 \mathrm{~Hz}, 11.4 \mathrm{~Hz}, 5-\mathrm{CH}(\mathrm{H})$ ), 2.47 (dddd, $1 \mathrm{H}, J=13.76 \mathrm{~Hz}, 4.40 \mathrm{~Hz}, 2.0 \mathrm{~Hz}, 2.0 \mathrm{~Hz}, 5-\mathrm{CH}(H)$ ), 3.28 (dd, 1H, $J=10.59 \mathrm{~Hz}, 7.3$ Hz, CH(H)I), 3.43 (dd, $1 \mathrm{H}, J=10.59 \mathrm{~Hz}, 4.5 \mathrm{~Hz}, \mathrm{CH}(H) \mathrm{I}), 3.82$ (s, 3H, $\mathrm{CH}_{3} \mathrm{O}$ ), 4.41 (dddd, $1 \mathrm{H}, J=11.28 \mathrm{~Hz}, 7.1 \mathrm{~Hz}, 4.6 \mathrm{~Hz}, 2.0 \mathrm{~Hz}, \mathrm{CHO}), 4.57$ (dd, $1 \mathrm{H}, J=11.56 \mathrm{~Hz}, 4.40 \mathrm{~Hz}, \mathrm{CHN}$ ), 5.31 (br s, 1H, NH), 6.89-6.94 (m, 2H, Ar-H), 7.23-7.28 (m, 2H, Ar-H). ${ }^{13} \mathrm{C}$ NMR (75 MHz, $\left.\mathrm{CDCl}_{3}\right): \delta=5.7,37.0,54.6,55.5,76.0,114.6,127.5,132.1,153.3,160.0$. IR $\left(\mathrm{ATR}, \mathrm{cm}^{-1}\right): v=$ $3249(\mathrm{NH}), 3113,1682(\mathrm{C}=\mathrm{O})$. MS (ES, $\mathrm{M}+\mathrm{H}^{+}$, pos): m/z $348\left(\mathrm{M}+\mathrm{H}^{+}, 100\right)$. Anal. Calcd. for $\mathrm{C}_{12} \mathrm{H}_{14} \mathrm{INO}_{3}$ : C, 41.52; H, 4.06; N, 4.03. Found: C, 41.56; H, 3.75; N, 4.01.

4.2.10. trans-6-(Iodomethyl)-4-(4-methoxyphenyl)-1,3-oxazinan-2-one (10d). Isolated by column chromatography (diethyl ether). Amorphous white solid; mp: 157.2-159.2 ${ }^{\circ} \mathrm{C}$; yield 7\%. ${ }^{1} \mathrm{H}$ NMR (300 MHz, $\mathrm{CDCl}_{3}$ ): $\delta=2.16$ (dddd, $1 \mathrm{H}, J=13.9 \mathrm{~Hz}, 4.2 \mathrm{~Hz}, 3.1 \mathrm{~Hz}, 0.9 \mathrm{~Hz}, 5-$ $\mathrm{CH}(\mathrm{H})$ ), 2.33 (ddd, 1H, $J=14.1 \mathrm{~Hz}, 8.9 \mathrm{~Hz}, 5.4 \mathrm{~Hz}, 5-\mathrm{CH}(H)$ ), 3.30 (dd, $1 \mathrm{H}, J=10.73 \mathrm{~Hz}$, $7.15 \mathrm{~Hz}, \mathrm{CH}(\mathrm{H}) \mathrm{I}), 3.36$ (dd, $1 \mathrm{H}, J=10.73 \mathrm{~Hz}, 4.95 \mathrm{~Hz}, \mathrm{CH}(H) \mathrm{I}), 3.82$ (s, 3H, $\mathrm{CH}_{3} \mathrm{O}$ ), 4.25 (dddd, 1H, $J=9.1 \mathrm{~Hz}, 7.15 \mathrm{~Hz}, 4.95 \mathrm{~Hz}, 3.03 \mathrm{~Hz}, \mathrm{CHO}$ ), 4.69-4.73 (m, 1H, CHN), 5.54 (br s, 1H, NH), 6.90-6.95 (m, 2H, Ar-H), 7.20-7.25 (m, 2H, Ar-H). ${ }^{13} \mathrm{C}$ NMR (75 MHz, $\left.\mathrm{CDCl}_{3}\right): \delta$ $=5.3$, 34.5, 51.7, 55.5, 72.8, 114.5, 127.0, 133.1, 153.3, 159.6. IR $\left(\right.$ ATR, $\left.\mathrm{cm}^{-1}\right): v=3245$ (NH), $1664(\mathrm{C}=\mathrm{O})$. MS (ES, $\mathrm{M}+\mathrm{H}^{+}$, pos): $\mathrm{m} / \mathrm{z} 348\left(\mathrm{M}+\mathrm{H}^{+}, 100\right)$. Anal. Calcd. for $\mathrm{C}_{12} \mathrm{H}_{14} \mathrm{INO}_{3}$ : C, 41.52; H, 4.06; N, 4.03. Found: C, 41.52; H, 3.70; N, 3.94.

4.2.11. 4-Phenyl-6-(phenylselanylmethyl)-1,3-oxazinan-2-one (10e). Mixture of cis- and trans-isomer in a ratio 54/46, crystallized from diethyl ether/hexane. Amorphous white solid; mp: 127.4-129.4 ${ }^{\circ} \mathrm{C}$; yield 79\%. ${ }^{1} \mathrm{H}$ NMR (300 $\left.\mathrm{MHz} \mathrm{CDCl}_{3}\right): \delta=1.75$ (dt, $1 \mathrm{H}, J=13.76 \mathrm{~Hz}$, $11.56 \mathrm{~Hz}, 5-\mathrm{CH}(\mathrm{H})_{\text {cis }}$ ), 2.23-2.27 (m, 2H, 5-CH(H) trans), 2.51 (ddt, $1 \mathrm{H}, J=13.76 \mathrm{~Hz}, 4.68 \mathrm{~Hz}$, $\left.1.8 \mathrm{~Hz}, 5-\mathrm{CH}(H)_{c i s}\right), 2.95$ (dd, $1 \mathrm{H}, J=12.80 \mathrm{~Hz}, 9.2 \mathrm{~Hz}, \mathrm{CH}(\mathrm{H}) \mathrm{Se}_{\text {trans }}$ ), 2.98 (dd, $1 \mathrm{H}, J=$ $12.93 \mathrm{~Hz}, 8.53 \mathrm{~Hz}, \mathrm{CH}(\mathrm{H}) \mathrm{Se}_{\text {cis }}$ ), 3.24 (dd, $1 \mathrm{H}, J=12.93 \mathrm{~Hz}, 4.68 \mathrm{~Hz}, \mathrm{CH}(H) \mathrm{Se}_{\text {trans }}$ ), 3.33 (dd, $1 \mathrm{H}, J=12.93 \mathrm{~Hz}, 4.68 \mathrm{~Hz}, \mathrm{CH}(H) \mathrm{Se}_{\text {cis }}$ ), 4.24-4.34 (m, $1 \mathrm{H}, \mathrm{CHO}_{\text {trans }}$ ), 4.50 (dddd, $1 \mathrm{H}, J=$ $11.39 \mathrm{~Hz}, 8.6 \mathrm{~Hz}, 4.7 \mathrm{~Hz}, 2.0 \mathrm{~Hz}, \mathrm{CHO}_{\text {cis }}$ ), 4.56 (dd, $1 \mathrm{H}, J=11.42 \mathrm{~Hz}, 4.5 \mathrm{~Hz}, \mathrm{CHN}_{c i s}$ ), 4.644.68 (m, $1 \mathrm{H}, \mathrm{CHN}_{\text {trans }}$ ), 5.32 (br s, $1 \mathrm{H}, \mathrm{NH}_{\text {cis }}$ ), 5.60 (br s, $1 \mathrm{H}, \mathrm{NH}_{\text {trans }}$ ), 7.16-7.54 (m, 20H, Ar$\mathrm{H}_{\text {cisttrans }}$ ). ${ }^{13} \mathrm{C} \mathrm{NMR}\left(75 \mathrm{MHz}, \mathrm{CDCl}_{3}\right): \delta=30.6,31.2,33.4,36.2,52.6,55.6,73.5,76.7$ (overlap with signal from $\mathrm{CDCl}_{3}$ ), 125.9, 126.2, 127.7, 127.9, 128.2, 128.8, 129.1, 129.3, 
129.4, 129.5, 132.9, 134.0, 140.5, 141.5, 153.6, 153.7. IR (ATR, $\left.\mathrm{cm}^{-1}\right): v=3211(\mathrm{NH}), 3109$, $1686(\mathrm{C}=\mathrm{O})$. MS (ES, $\mathrm{M}+\mathrm{H}^{+}$, pos): m/z 348/346 (M+H', 100). Anal. Calcd. for $\mathrm{C}_{17} \mathrm{H}_{17} \mathrm{NO}_{2} \mathrm{Se}$ : C, 58.96; H, 4.95; N, 4.04. Found: C, 59.08; H, 4.56; N, 4.02.

4.2.12. 4-(Methoxyphenyl)-6-(phenylselanylmethyl)-1,3-oxazinan-2-one (10f). Mixture of cis- and trans-isomer in a ratio 58/42, crystallized from diethyl ether/hexane. Amorphous white solid; mp: 93.1-95.1 ${ }^{\circ} \mathrm{C}$; yield 85\%. ${ }^{1} \mathrm{H}$ NMR (300 MHz, $\left.\mathrm{CDCl}_{3}\right): \delta=1.74(\mathrm{dt}, 1 \mathrm{H}, J=$ $13.76 \mathrm{~Hz}, 11.56 \mathrm{~Hz}, 5-\mathrm{CH}(\mathrm{H})_{\text {cis }}$ ), 2.18-2.24 (m, 2H, 5-CH(H) trans), 2.47 (ddt, $1 \mathrm{H}, J=13.76 \mathrm{~Hz}$, $\left.4.40 \mathrm{~Hz}, 1.9 \mathrm{~Hz}, 5-\mathrm{CH}(H)_{c i s}\right), 2.97$ (dd, 1H, $J=12.80 \mathrm{~Hz}, 9.2 \mathrm{~Hz}, \mathrm{CH}(\mathrm{H}) \mathrm{Se}_{\text {trans }}$ ), 2.98 (dd, 1H, $J=13.07 \mathrm{~Hz}, 8.4 \mathrm{~Hz}, \mathrm{CH}(\mathrm{H}) \mathrm{Se}_{\text {cis }}$ ), 3.26 (dd, $\left.1 \mathrm{H}, J=12.80 \mathrm{~Hz}, 4.5 \mathrm{~Hz}, \mathrm{CH}(H) \mathrm{Se}_{\text {trans }}\right)$, 3.33 (dd, $1 \mathrm{H}, J=12.93 \mathrm{~Hz}, 4.68 \mathrm{~Hz}, \mathrm{CH}(H) \mathrm{Se}_{\text {cis }}$ ), 3.81 (s, $3 \mathrm{H}, \mathrm{CH}_{3} \mathrm{O}_{\text {cis }}$ ), 3.82 (s, $3 \mathrm{H}, \mathrm{CH}_{3} \mathrm{O}_{\text {trans }}$ ), 4.27-4.35 (m, $1 \mathrm{H}, \mathrm{CHO}_{\text {trans }}$ ), 4.44-4.53 (m, $1 \mathrm{H}, \mathrm{CHO}_{\text {cis }}$ ), 4.51 (dd, $1 \mathrm{H}, J=11.28 \mathrm{~Hz}, 4.3 \mathrm{~Hz}$, $\mathrm{CHN}_{\text {cis }}$ ), 4.57-4.62 (m, $1 \mathrm{H}, \mathrm{CHN}_{\text {trans }}$ ), 5.15 (br s, $1 \mathrm{H}, \mathrm{NH}_{\text {cis }}$ ), 5.38 (br s, $1 \mathrm{H}, \mathrm{NH}_{\text {trans }}$ ), 6.867.54 (m, 18H, Ar- $\left.\left.\mathrm{H}_{\text {cis }+ \text { trans }}\right) .{ }^{13} \mathrm{C} \mathrm{NMR} \mathrm{(75} \mathrm{MHz,} \mathrm{CDCl}_{3}\right): \delta=30.6,31.3,33.4,36.3,51.9,55.0$, 55.5, 73.6, 76.6, 114.3, 114.5, 127.1, 127.4, 127.6, 127.9, 128.6, 129.2, 129.4, 129.5, 132.4, 132.8, 133.5, 133.9, 153.8, 153.9, 159.4, 159.9. IR (ATR, $\left.\mathrm{cm}^{-1}\right): v=3225(\mathrm{NH}), 3102,1685$

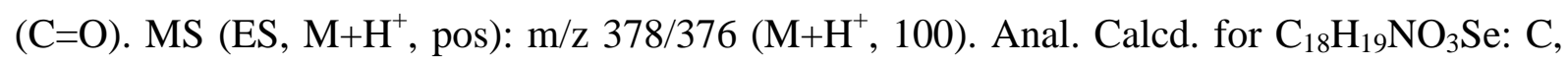
57.45; H, 5.09; N, 3.72. Found: C, 57.46; H, 5.10; N, 3.72.

4.2.13. Alternative procedure for the preparation of 4-aryl-1,3-oxazinan-2-ones 10 . To a solution of homoallylamine $9(1 \mathrm{mmol})$ in dichloromethane $(20 \mathrm{~mL})$ at $0{ }^{\circ} \mathrm{C}$, TBDMSOTf (0.79 g, $3 \mathrm{mmol}$ ) and 2,6-lutidine (0.43 g, $4 \mathrm{mmol}$ ) was added. After stirring for $2 \mathrm{~h} \mathrm{at} 0{ }^{\circ} \mathrm{C}$, a solution of electrophile $(2.44 \mathrm{mmol})$ in freshly distilled dry dichloromethane $(10 \mathrm{~mL})$ was added dropwise and the mixture was stirred at $0{ }^{\circ} \mathrm{C}$ under nitrogen atmosphere for $16-24$ hours. After typical workup, the mixture was purified by column chromatography (EtOAc) to afford 4-aryl-1,3-oxazinan-2-ones $\mathbf{1 0}$ as a mixture of trans- and cis-isomers.

\subsubsection{General procedure for the preparation of benzyl $N$-benzyl- $N$-(1-phenyl-3-}

butenyl)carbamates 11. Benzyl bromide $(60 \mathrm{mmol})$ was added to a solution of sodium iodide in acetone $(80 \mathrm{~mL})$. The mixture was stirred for $24 \mathrm{~h}$ in the dark at room temperature, quenched with water $(50 \mathrm{~mL})$ and extracted with $\mathrm{Et}_{2} \mathrm{O}(2 \times 100 \mathrm{~mL})$. The combined organic layers were dried $\left(\mathrm{MgSO}_{4}\right)$, filtered and concentrated under reduced pressure to afford the pure benzyl iodide as a yellow oil. Sodium hydride $(5.25 \mathrm{mmol})$ was washed three times with $n$-pentane after which DMF (10 mL) was added. The suspension was cooled to $0{ }^{\circ} \mathrm{C}$ and a 
solution of homoallylamine 9 (3.5 mmol) dissolved in dry DMF $(8 \mathrm{~mL})$ was added. The mixture was stirred for 30 min and then transferred by cannula into a flask containing benzyl iodide (60 mmol) in dry DMF (15 mL). The mixture was stirred for additional $10 \mathrm{~min}$ at room temperature. The reaction was quenched with saturated aqueous $\mathrm{NH}_{4} \mathrm{Cl}$ solution and extracted with $\mathrm{Et}_{2} \mathrm{O}(2 \times 30 \mathrm{~mL})$. The combined organic layers were dried $\left(\mathrm{MgSO}_{4}\right)$, filtered and concentrated under reduced pressure. The residue was purified by flash chromatography (hexane/Et ${ }_{2} \mathrm{O}$ 6:1) to give compounds $\mathbf{1 1}$.

4.2.15. Benzyl $N$-benzyl- $N$-(1-phenyl-3-butenyl)carbamate (11a). Colourless oil; yield 70\%. ${ }^{1} \mathrm{H} \mathrm{NMR}\left(300 \mathrm{MHz}, \mathrm{CDCl}_{3}\right.$, recorded at $\left.50{ }^{\circ} \mathrm{C}\right): \delta=2.67\left(\mathrm{t}, 2 \mathrm{H}, J=7.2 \mathrm{~Hz}, \mathrm{CH}_{2} \mathrm{CH}=\right.$ ), 4.19 (d, 1H, $J=15.69$ Hz, NCH(H)), 4.42 (d, 1H, $J=15.69$ Hz, NCH(H)), 4.93-4.99 (m, 2H, $\left.=\mathrm{CH}_{2}\right), 5.17\left(\mathrm{~s}, 2 \mathrm{H}, \mathrm{OCH}_{2}\right), 5.32-5.43(\mathrm{~m}, 1 \mathrm{H}, \mathrm{CHN}), 5.60-5.73(\mathrm{~m}, 1 \mathrm{H}, \mathrm{CH}=)$, 7.00-7.33 (m, 15H, Ar-H). IR (ATR, $\left.\mathrm{cm}^{-1}\right): v=1691(\mathrm{C}=\mathrm{O})$. MS (ES, pos): $\mathrm{m} / \mathrm{z} 372\left(\mathrm{M}+\mathrm{H}^{+}, 100\right)$. Purity $>94 \%$ as determined by reverse phase HPLC analysis (integration at $220 \mathrm{~nm}$ ).

4.2.16. Benzyl $N$-benzyl- $N$-(1-(4-methoxyphenyl)-3-butenyl)carbamate (11b). Colourless oil; yield 65\%. ${ }^{1} \mathrm{H} \mathrm{NMR}\left(300 \mathrm{MHz}, \mathrm{CDCl}_{3}\right.$, recorded at $\left.50{ }^{\circ} \mathrm{C}\right): \delta=2.63$ (t, $2 \mathrm{H}, J=7.2 \mathrm{~Hz}$, $\left.\mathrm{CH}_{2} \mathrm{CH}=\right), 3.78$ (s, 3H, $\mathrm{CH}_{3} \mathrm{O}$ ), 4.16 (d, $\left.1 \mathrm{H}, J=15.96 \mathrm{~Hz}, \mathrm{NCH}(\mathrm{H})\right), 4.40$ (d, $1 \mathrm{H}, J=15.69$ $\mathrm{Hz}, \mathrm{NCH}(H)$ ), 4.92-4.98 (m, 2H, = $\mathrm{CH}_{2}$ ), 5.17 (s, 2H, $\mathrm{OCH}_{2}$ ), 5.28-5.38 (m, 1H, CHN), 5.59$5.72(\mathrm{~m}, 1 \mathrm{H}, \mathrm{CH}=)$, 6.77-6.82 (m, 2H, Ar-H), 7.00-7.32 (m, 12H, Ar-H). ${ }^{13} \mathrm{C}$ NMR (75 MHz, $\mathrm{CDCl}_{3}$ ): $\delta=36.1,47.2$ (broad), 55.4, 58.9, 67.4, 113.8, 117.5, 126.9, 127.3 (broad), 128.0 (broad), 128.2, 128.5, 129.6, 131.4, 135.0, 136.7, 139.1, 156.9, 159.1. IR (ATR, $\left.\mathrm{cm}^{-1}\right): v=$

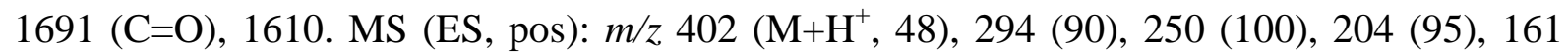
(72). Purity $>92 \%$ as determined by reverse phase HPLC analysis (integration at $220 \mathrm{~nm}$ ).

\subsubsection{General procedure for the preparation of 4-aryl-3-benzyl-1,3-oxazinan-2-ones 12 .}

A solution of electrophile $(1.22 \mathrm{mmol})$ in freshly distilled dry dichloromethane $(10 \mathrm{~mL})$ was added dropwise in a period of 10 minutes to a stirred solution of benzyl $N$-benzyl- $N$-(1phenyl-3-butenyl)carbamate $11(1.0 \mathrm{mmol})$ in dichloromethane $(20 \mathrm{~mL})$ and the mixture was stirred at room temperature under nitrogen atmosphere for 18 - 40 hours. After typical workup, the mixture was purified by column chromatography (EtOAc) to afford 4-aryl-3benzyl-1,3-oxazinan-2-ones 12 as a mixture of trans- and cis-isomers. 
4.2.18. trans-3-Benzyl-6-(bromomethyl)-4-phenyl-1,3-oxazinan-2-one (12a). Isolated by column chromatography (EtOAc) and crystallization from dichloromethane/hexane. Amorphous white solid; mp: 131.7-133.7 ${ }^{\circ} \mathrm{C}$; yield 86\%. ${ }^{1} \mathrm{H}$ NMR (300 $\left.\mathrm{MHz}, \mathrm{CDCl}_{3}\right): \delta=$ 2.13 (ddd, 1H, $J=13.76 \mathrm{~Hz}, 2.75 \mathrm{~Hz}, 2.75 \mathrm{~Hz}, 5-\mathrm{CH}(\mathrm{H})$ ), 2.20 (ddd, 1H, $J=13.69 \mathrm{~Hz}, 10.9$ Hz, $5.6 \mathrm{~Hz}, 5-\mathrm{CH}(H)$ ), 3.42 (dd, $1 \mathrm{H}, J=10.87 \mathrm{~Hz}, 6.2 \mathrm{~Hz}, \mathrm{CH}(\mathrm{H}) \mathrm{Br}$ ), 3.47 (dd, $1 \mathrm{H}, J=10.87$ Hz, 4.5 Hz, CH(H)Br), 3.66 (d, 1H, $J=15.13$ Hz, NCH(H)), 4.36-4.44 (m, 1H, CHO), 4.53 (dd, 1H, $J=5.50 \mathrm{~Hz}, 2.48 \mathrm{~Hz}, \mathrm{CHN}$ ), 5.40 (d, 1H, $J=15.13 \mathrm{~Hz}, \mathrm{NCH}(H)$ ), 7.20-7.47 (m, $\left.10 \mathrm{H}, 2 \mathrm{xC}_{6} \mathrm{H}_{5}\right) .{ }^{13} \mathrm{C} \mathrm{NMR}\left(75 \mathrm{MHz}, \mathrm{CDCl}_{3}\right): \delta=33.0,34.2,50.6,55.8,71.6,126.3,128.0$, 128.3, 128.4, 128.9, 129.4, 136.5, 139.5, 153.6. IR (ATR, $\left.\mathrm{cm}^{-1}\right): v=1674(\mathrm{C}=\mathrm{O})$. MS (ES, pos): m/z 360/362 (M+H', 100). Anal. Calcd. for $\mathrm{C}_{18} \mathrm{H}_{18} \mathrm{BrNO}_{2}$ : C, 60.01; H, 5.04; N, 3.89 . Found: C, 59.94; H, 4.64; N, 3.83.

4.2.19. trans-3-Benzyl-6-(bromomethyl)-4-(4-methoxyphenyl)-1,3-oxazinan-2-one (12b). Isolated together with the cis-isomer $\mathbf{1 2 b}$ as 85:15 mixture by column chromatography (EtOAc). Viscous colourless oil; yield 85\%. ${ }^{1} \mathrm{H}$ NMR (300 MHz, $\mathrm{CDCl}_{3}$ ): $\delta=2.09$ (ddd, $1 \mathrm{H}$, $J=13.7 \mathrm{~Hz}, 2.8 \mathrm{~Hz}, 2.8 \mathrm{~Hz}$, 5-CH(H)), 2.16 (ddd, 1H, $J=13.7 \mathrm{~Hz}, 11.01 \mathrm{~Hz}, 5.6 \mathrm{~Hz}, 5-$ $\mathrm{CH}(H)$ ), 3.41 (dd, $1 \mathrm{H}, J=10.8 \mathrm{~Hz}, 6.33 \mathrm{~Hz}, \mathrm{CH}(\mathrm{H}) \mathrm{Br}$ ), 3.47 (dd, $1 \mathrm{H}, J=10.8 \mathrm{~Hz}, 4.68 \mathrm{~Hz}$, $\mathrm{CH}(\mathrm{H}) \mathrm{Br}$ ), 3.84 (s, 3H, $\mathrm{CH}_{3} \mathrm{O}$ ), 3.65 (d, 1H, $J=15.13 \mathrm{~Hz}, \mathrm{NCH}(\mathrm{H})$ ), 4.37-4.45 (m, 1H, CHO), 4.47 (dd, $1 \mathrm{H}, J=5.23 \mathrm{~Hz}, 2.20 \mathrm{~Hz}, \mathrm{CHN}$ ), 5.37 (d, 1H, $J=15.13 \mathrm{~Hz}, \mathrm{NCH}(H)$ ), 6.92-7.37 (m, 9H, Ar-H). ${ }^{13} \mathrm{C}$ NMR (75 MHz, $\left.\mathrm{CDCl}_{3}\right): \delta=33.0,34.3$, 50.5, 55.3, 55.5, 71.7, 114.7, 127.5, 127.9, 128.3, 128.9, 131.3, 136.5, 153.6, 159.6. IR (ATR, $\left.\mathrm{cm}^{-1}\right): v=1689(\mathrm{C}=\mathrm{O})$. MS (ES, pos): $\mathrm{m} / \mathrm{z}$ 390/392 $\left(\mathrm{M}+\mathrm{H}^{+}, 100\right)$. Purity $>90 \%$ as determined by reverse phase HPLC analysis (integration at $220 \mathrm{~nm}$ ).

4.2.20. trans-3-Benzyl-6-(iodomethyl)-4-phenyl-1,3-oxazinan-2-one (12c). Isolated together with the cis-isomer 12c as 94:6 mixture by column chromatography (EtOAc) and crystallization from dichloromethane/hexane. Amorphous white solid; mp: 135.8-137.8 ${ }^{\circ} \mathrm{C}$; yield 85\%. ${ }^{1} \mathrm{H}$ NMR (300 MHz, $\left.\mathrm{CDCl}_{3}\right): \delta=2.07-2.21\left(\mathrm{~m}, 2 \mathrm{H}, 5-\mathrm{CH}_{2}\right), 3.23(\mathrm{dd}, 1 \mathrm{H}, J=$ $10.59 \mathrm{~Hz}, 6.5 \mathrm{~Hz}, \mathrm{CH}(\mathrm{H}) \mathrm{I}), 3.30$ (dd, 1H, $J=10.59 \mathrm{~Hz}, 4.8 \mathrm{~Hz}, \mathrm{CH}(H) \mathrm{I}), 3.65$ (d, 1H, $J=$ $15.13 \mathrm{~Hz}, \mathrm{NCH}(\mathrm{H})), 4.14-4.22$ (m, 1H, CHO), 4.51 (dd, $1 \mathrm{H}, J=5.23 \mathrm{~Hz}, 2.75 \mathrm{~Hz}, \mathrm{CHN}$ ), $5.40(\mathrm{~d}, 1 \mathrm{H}, J=15.13 \mathrm{~Hz}, \mathrm{NCH}(H)), 7.18-7.46\left(\mathrm{~m}, 10 \mathrm{H}, 2 \mathrm{xC}_{6} \mathrm{H}_{5}\right) .{ }^{13} \mathrm{C} \mathrm{NMR}(75 \mathrm{MHz}$, $\left.\mathrm{CDCl}_{3}\right): \delta=6.3,35.9,50.5,55.8,71.8,126.3,127.9,128.3,128.4,128.9,129.4,136.5,139.6$, 
153.7. IR (ATR, $\left.\mathrm{cm}^{-1}\right): v=1677(\mathrm{C}=\mathrm{O})$. MS (ES, pos): m/z $408\left(\mathrm{M}+\mathrm{H}^{+}, 100\right)$. Anal. Calcd. for $\mathrm{C}_{18} \mathrm{H}_{18} \mathrm{INO}_{2}$ : C, 53.09; H, 4.46; N, 3.44. Found: C, 52.73; H, 4.16; N, 3.62.

4.2.21. trans-3-Benzyl-6-(iodomethyl)-4-(4-methoxyphenyl)-1,3-oxazinan-2-one (12d). Isolated together with the cis-isomer 12d as 91:9 mixture by column chromatography (EtOAc). Viscous yellow oil; yield 86\%. ${ }^{1} \mathrm{H}$ NMR (300 MHz, $\left.\mathrm{CDCl}_{3}\right): \delta=2.04-2.18(\mathrm{~m}, 2 \mathrm{H}$, 5- $\mathrm{CH}_{2}$ ), 3.23 (dd, 1H, $\left.J=10.46 \mathrm{~Hz}, 6.60 \mathrm{~Hz}, \mathrm{CH}(\mathrm{H}) \mathrm{I}\right), 3.30$ (dd, 1H, $J=10.46 \mathrm{~Hz}, 4.68 \mathrm{~Hz}$, $\mathrm{CH}(H) \mathrm{I}), 3.65$ (d, 1H, $J=14.86 \mathrm{~Hz}, \mathrm{NCH}(\mathrm{H})$ ), 3.84 (s, 3H, $\left.\mathrm{CH}_{3} \mathrm{O}\right), 4.15-4.23$ (m, 1H, CHO), 4.45 (dd, 1H, $J=4.95 \mathrm{~Hz}, 2.75 \mathrm{~Hz}, \mathrm{CHN}$ ), 5.38 (d, 1H, $J=15.13 \mathrm{~Hz}, \mathrm{NCH}(H)$ ), 6.92-7.37 (m, 9H, Ar-H). ${ }^{13} \mathrm{C}$ NMR (75 MHz, $\mathrm{CDCl}_{3}$ ): $\delta=6.2,36.0,50.4,55.3,55.5,71.9,114.7,127.4$, 127.9, 128.3, 128.9, 131.4, 136.6, 139.6, 153.6, 159.6. IR $\left(\mathrm{ATR}, \mathrm{cm}^{-1}\right): v=1687(\mathrm{C}=\mathrm{O}) . \mathrm{MS}$ (ES, pos): m/z $438\left(\mathrm{M}+\mathrm{H}^{+}\right.$, 100). Anal. Calcd. for $\mathrm{C}_{19} \mathrm{H}_{20} \mathrm{INO}_{3}$ : C, 52.19; H, 4.61; N, 3.20. Found: C, 52.53; H, 4.83; N, 3.44.

4.2.22. 3-Benzyl-6-(phenylselanylmethyl)-4-phenyl-1,3-oxazinan-2-one (12e). Isolated as a 66:33 trans/cis mixture by column chromatography (EtOAc). Viscous colourless oil; yield 78\%. ${ }^{1} \mathrm{H}$ NMR (300 MHz, $\mathrm{CDCl}_{3}$ ): $\delta=1.93\left(\mathrm{dt}, 1 \mathrm{H}, J=14.03 \mathrm{~Hz}, 11.4 \mathrm{~Hz}, 5-\mathrm{CH}(\mathrm{H})_{\text {cis }}\right.$ ), 2.06 (ddd, $1 \mathrm{H}, J=13.76 \mathrm{~Hz}, 10.7 \mathrm{~Hz}, 5.8 \mathrm{~Hz}, 5-\mathrm{CH}(\mathrm{H})_{\text {trans }}$ ), 2.27 (dt, 1H, $J=13.76 \mathrm{~Hz}, 2.48 \mathrm{~Hz}$, 5-CH(H) trans), 2.52 (ddd, 1H, $J=13.97 \mathrm{~Hz}, 5.9 \mathrm{~Hz}, 1.9 \mathrm{~Hz}, 5-\mathrm{CH}(H)_{\text {cis }}$ ), 2.85 (dd, 1H, $J=$ $12.66 \mathrm{~Hz}, 8.81 \mathrm{~Hz}, \mathrm{CH}(\mathrm{H}) \mathrm{Se}_{\text {trans }}$ ), 2.94 (dd, $1 \mathrm{H}, J=12.93 \mathrm{~Hz}, 8.26 \mathrm{~Hz}, \mathrm{CH}(\mathrm{H}) \mathrm{Se}_{\text {cis }}$ ), 3.20 (dd, $1 \mathrm{H}, J=12.93 \mathrm{~Hz}, 4.40 \mathrm{~Hz}, \mathrm{CH}(H) \mathrm{Se}_{\text {trans }}$ ), 3.30 (dd, $1 \mathrm{H}, J=12.93 \mathrm{~Hz}, 4.68 \mathrm{~Hz}, \mathrm{CH}(H) \mathrm{Se}_{\text {cis }}$ ), 3.54 (d, 1H, $J=15.13 \mathrm{~Hz}, \mathrm{NCH}(\mathrm{H})_{c i s}$ ), 3.60 (d, 1H, $J=15.13 \mathrm{~Hz}, \mathrm{NCH}(\mathrm{H})_{\text {trans }}$ ), 4.22-4.38 (m, $3 \mathrm{H}, \mathrm{CHO}_{\text {trans }+ \text { cis }}$ and $\mathrm{CHN}_{\text {cis }}$ ), 4.43 (dd, $1 \mathrm{H}, J=5.78 \mathrm{~Hz}, 2.20 \mathrm{~Hz}, \mathrm{CHN}_{\text {trans }}$ ), 5.22 (d, $1 \mathrm{H}, J=$ $\left.14.86 \mathrm{~Hz}, \mathrm{NCH}(H)_{\text {cis }}\right), 5.36$ (d, $\left.1 \mathrm{H}, J=15.13 \mathrm{~Hz}, \mathrm{NCH}(H)_{\text {trans }}\right)$, 7.03-7.51 (m, 30H, Ar$\mathrm{H}_{\text {cistrans }}$ ). ${ }^{13} \mathrm{C}$ NMR (75 MHz, $\mathrm{CDCl}_{3}$ ): $\delta=31.0,31.1$, 34.8, 38.0, 48.9, 50.4, 56.1, 58.6, 72.6, 74.9, 126.4, 127.1, 127.6, 127.7, 127.8, 128.1, 128.3, 128.5, 128.6, 128.7, 128.8, 129.2, 129.3, 129.5, 132.8, 134.1, 136.7, 136.8, 139.8, 139.9, 154.2, 154.8. IR (ATR, $\left.\mathrm{cm}^{-1}\right): v=1688$ (C=O). MS (ES, pos): $m / z$ 436/438 $\left(\mathrm{M}+\mathrm{H}^{+}, 100\right)$. Anal. Calcd. for $\mathrm{C}_{24} \mathrm{H}_{23} \mathrm{NO}_{2} \mathrm{Se}: \mathrm{C}, 66.05 ; \mathrm{H}$, 5.31; N, 3.21. Found: C, 65.67; H, 5.58; N, 3.52.

\subsubsection{3-Benzyl-4-(4-methoxyphenyl)-6-(phenylselanylmethyl)-1,3-oxazinan-2-one (12f).} Isolated as a 50:50 trans/cis mixture by column chromatography (EtOAc). Viscous yellow oil; yield 82\%. ${ }^{1} \mathrm{H}$ NMR (300 MHz, $\mathrm{CDCl}_{3}$ ): $\delta=1.92$ (dt, $1 \mathrm{H}, J=14.31 \mathrm{~Hz}, 11.5 \mathrm{~Hz}, 5-\mathrm{CH}(\mathrm{H})_{c i s}$ ), 
2.02 (ddd, $1 \mathrm{H}, J=13.62 \mathrm{~Hz}, 10.87 \mathrm{~Hz}, 5.6 \mathrm{~Hz}, 5-\mathrm{CH}(\mathrm{H})_{\text {trans }}$ ), 2.22 (dt, $1 \mathrm{H}, J=13.48 \mathrm{~Hz}, 2.48$ $\mathrm{Hz}, 5-\mathrm{CH}(H)_{\text {trans }}$ ), 2.49 (ddd, $1 \mathrm{H}, J=13.97 \mathrm{~Hz}, 6.1 \mathrm{~Hz}, 1.6 \mathrm{~Hz}, 5-\mathrm{CH}(H)_{\text {cis }}$ ), 2.85 (dd, $1 \mathrm{H}, J=$ $12.80 \mathrm{~Hz}, 8.9 \mathrm{~Hz}, \mathrm{CH}(\mathrm{H}) \mathrm{Se}_{\text {trans }}$ ), 2.95 (dd, 1H, J = 12.93 Hz, 8.53 Hz, CH(H)Se $\mathrm{C}_{\text {cis }}$ ), 3.21 (dd, $1 \mathrm{H}, J=12.80 \mathrm{~Hz}, 4.5 \mathrm{~Hz}, \mathrm{CH}(H) \mathrm{Se}_{\text {trans }}$ ), 3.30 (dd, $1 \mathrm{H}, J=12.93 \mathrm{~Hz}, 4.68 \mathrm{~Hz}, \mathrm{CH}(H) \mathrm{Se}_{\text {cis }}$ ), 3.55 (d, 1H, $J=15.13 \mathrm{~Hz}, \mathrm{NCH}(\mathrm{H})_{\text {cis }}$ ), 3.60 (d, 1H, $J=15.13 \mathrm{~Hz}, \mathrm{NCH}(\mathrm{H})_{\text {trans }}$ ), 3.83 (s, 3H, $\mathrm{CH}_{3} \mathrm{O}_{\text {cis }}$ ), 3.85 (s, $3 \mathrm{H}, \mathrm{CH}_{3} \mathrm{O}_{\text {trans }}$ ), 4.21-4.34 (m, 3H, $\mathrm{CHO}_{\text {trans+cis }}$ and $\mathrm{CHN}_{\text {cis }}$ ), 4.37 (dd, $1 \mathrm{H}, J$ $\left.=5.50 \mathrm{~Hz}, 2.75 \mathrm{~Hz}, \mathrm{CHN}_{\text {trans }}\right), 5.19$ (d, $\left.1 \mathrm{H}, J=15.13 \mathrm{~Hz}, \mathrm{NCH}(H)_{\text {cis }}\right), 5.34$ (d, $1 \mathrm{H}, J=15.13$ $\left.\mathrm{Hz}, \mathrm{NCH}(H)_{\text {trans }}\right), 6.88-7.51$ (m, 28H, Ar- $\left.\mathrm{H}_{\text {cisttrans }}\right) .{ }^{13} \mathrm{C}$ NMR $\left(75 \mathrm{MHz}, \mathrm{CDCl}_{3}\right): \delta=31.1$ (2C), 34.9, 38.0, 48.7, 50.3, 55.5 (2C), 55.6, 58.1, 72.6, 74.9, 114.5, 127.5, 127.6, 127.77, 127.80, 128.3, 128.4, 128.7, 128.8, 129.3, 129.4, 131.6, 131.8, 132.8, 134.0, 136.8, 136.9, 154.2, 154.8, 159.4, 159.7. IR (ATR, $\left.\mathrm{cm}^{-1}\right): v=1687(\mathrm{C}=\mathrm{O})$. MS (ES, pos): $\mathrm{m} / \mathrm{z}$ 466/468 $\left(\mathrm{M}+\mathrm{H}^{+}, 100\right)$. Anal. Calcd. for $\mathrm{C}_{25} \mathrm{H}_{25} \mathrm{NO}_{3}$ Se: C, 64.38; H, 5.40; N, 3.00. Found: C, 64.70; H, 5.69; N, 3.38 .

4.2.24. General procedure for the synthesis of 6-arylpiperidine-2,4-diones 15. To a solution of 4-aryl-6-(iodomethyl)-1,3-oxazinan-2-one 10 (1 mmol) in ethanol (25 mL) was added a $5 \mathrm{M}$ aqueous solution of $\mathrm{NaOH}(25 \mathrm{~mL})$ and the reaction mixture was stirred under reflux for $8 \mathrm{~h}$. After completion of the reaction, 5\% $\mathrm{HCl}$ (aq.) was added and the reaction mixture was extracted with EtOAc and $\mathrm{CH}_{2} \mathrm{Cl}_{2}$. The combined organic layers were dried $\left(\mathrm{MgSO}_{4}\right)$, filtered and concentrated under reduced pressure. The residue was crystallized from $\mathrm{CH}_{2} \mathrm{Cl}_{2}$ /hexane to afford the pure 6-arylpiperidine-2,4-diones 15 .

4.2.25. 6-Phenylpiperidine-2,4-dione 15a. Amorphous yellowish solid; mp: $166.2-168.2{ }^{\circ} \mathrm{C}$ (lit. mp: $167-169{ }^{\circ} \mathrm{C},{ }^{27} 166-168{ }^{\circ} \mathrm{C}$ for $(R)-(+)-15 a^{29}, 164-168{ }^{\circ} \mathrm{C}^{32}$ ); yield $86 \%$. ${ }^{1} \mathrm{H}$ NMR $\left(300 \mathrm{MHz}, \mathrm{CDCl}_{3}\right): \delta=2.77(\mathrm{dd}, 1 \mathrm{H}, J=16.0 \mathrm{~Hz}, 9.5 \mathrm{~Hz}, 5-\mathrm{CH}(\mathrm{H})$ ), 2.90 (dd, $1 \mathrm{H}, J=16.0$ Hz, 4.40 Hz, 5-CH(H)), 3.39 (s, 2H, 3- $\mathrm{CH}_{2}$ ), 4.82 (ddd, 1H, J = 9.56 Hz, $4.5 \mathrm{~Hz}, 1.7 \mathrm{~Hz}, \mathrm{CH}$ ), 6.25 (br s, $1 \mathrm{H}, \mathrm{NH}), 7.31-7.47$ (m, 5H, $\left.\mathrm{C}_{6} \mathrm{H}_{5}\right) .{ }^{13} \mathrm{C}$ NMR $\left(75 \mathrm{MHz}, \mathrm{CDCl}_{3}\right): \delta=47.1$, 47.3, 52.9, 126.1, 128.9, 129.5, 139.3, 169.3, 202.4. IR (ATR, $\left.\mathrm{cm}^{-1}\right): v=3185(\mathrm{NH}), 1715(\mathrm{C}=\mathrm{O})$, $1667(\mathrm{NC}=\mathrm{O})$. MS (ES, pos): m/z $190\left(\mathrm{M}+\mathrm{H}^{+}, 100\right)$. All spectroscopic data are in good correspondence with reported data. ${ }^{27,29,32}$

4.2.26. 6-(4-Methoxyphenyl)piperidine-2,4-dione 15b. Amorphous white solid; mp: 172.4174.4 ${ }^{\circ} \mathrm{C}$ (lit. mp: $174-175{ }^{\circ} \mathrm{C},{ }^{27} 171-172{ }^{\circ} \mathrm{C}^{43}$ ); yield 82\%. ${ }^{1} \mathrm{H}$ NMR $\left(300 \mathrm{MHz}, \mathrm{CDCl}_{3}\right): \delta=$ 
2.74 (dd, 1H, $J=16.1 \mathrm{~Hz}, 9.36 \mathrm{~Hz}, 5-\mathrm{CH}(\mathrm{H})$ ), 2.85 (dd, 1H, $J=16.1 \mathrm{~Hz}, 4.40 \mathrm{~Hz}, 5-\mathrm{CH}(H)$ ), 3.36 (s, 2H, 3- $\mathrm{CH}_{2}$ ), 3.82 (s, 3H, $\mathrm{OCH}_{3}$ ), 4.76 (dd, $1 \mathrm{H}, J=9.36 \mathrm{~Hz}, 4.40 \mathrm{~Hz}, \mathrm{CH}$ ), 6.37 (br s, 1H, NH), 6.93 (d, 2H, $J=8.81 \mathrm{~Hz}, \mathrm{Ar}-\mathrm{H}), 7.24$ (d, $2 \mathrm{H}, J=8.81 \mathrm{~Hz}, \mathrm{Ar}-\mathrm{H}) .{ }^{13} \mathrm{C}$ NMR $(75$ $\mathrm{MHz}, \mathrm{CDCl}_{3}$ ): $\delta=47.29,47.33,52.5,55.5,114.8,127.4,131.1,160.0,168.8,202.5$. IR (ATR, $\left.\mathrm{cm}^{-1}\right): v=3183(\mathrm{NH}), 1722(\mathrm{C}=\mathrm{O}), 1665(\mathrm{NC}=\mathrm{O})$. MS (ES, pos): m/z $220\left(\mathrm{M}+\mathrm{H}^{+}, 100\right)$.

\subsubsection{General procedure for the preparation of 6-(azidomethyl)-1,3-oxazinan-2-ones 17.}

To a solution of 6-(iodomethyl)-1,3-oxazinan-2-one 10 (1 mmol) in DMSO (10 mL) was added sodium azide ( $2 \mathrm{mmol}$ ) and the reaction mixture was stirred at $80{ }^{\circ} \mathrm{C}$ for $14 \mathrm{~h}$. After cooling, the reaction mixture was poured into $\mathrm{H}_{2} \mathrm{O}(30 \mathrm{~mL})$ and extracted with EtOAc $(3 \times 30$ $\mathrm{mL}$ ). The combined organic extracts were washed with brine. The organic layer was dried $\left(\mathrm{MgSO}_{4}\right)$, filtered and evaporated under reduced pressure. Crystallisation of the residue from $\mathrm{CH}_{2} \mathrm{Cl}_{2}$ /hexane afforded the pure 6-(azidomethyl)-1,3-oxazinan-2-ones $\mathbf{1 7}$ in 81-85\% yield.

4.2.28. 6-(Azidomethyl)-4-phenyl-1,3-oxazinan-2-one (17a). Mixture of cis- and transisomer in a ratio 69/31. Amorphous purple solid; mp: $122.7-125.7{ }^{\circ} \mathrm{C}$; yield $81 \%$. ${ }^{1} \mathrm{H}$ NMR (300 MHz, $\left.\mathrm{CDCl}_{3}\right): \delta=1.91\left(\mathrm{dt}, 1 \mathrm{H}, J=13.76 \mathrm{~Hz}, 11.83 \mathrm{~Hz}, 5-\mathrm{CH}(\mathrm{H})_{c i s}\right), 1.93-2.01(\mathrm{~m}, 1 \mathrm{H}$, 5-CH(H)trans), 2.17-2.24 (m, 1H, 5-CH(H) cis $_{\text {) }}, 2.36$ (ddd, $1 \mathrm{H}, J=13.8 \mathrm{~Hz}, 10.5 \mathrm{~Hz}, 5.8 \mathrm{~Hz}, 5-$ $\mathrm{CH}(H)_{\text {trans }}$ ), 3.43 (dd, $1 \mathrm{H}, J=13.07 \mathrm{~Hz}, 5.1 \mathrm{~Hz}, \mathrm{CH}(\mathrm{H}) \mathrm{N}_{3, \text { trans }}$ ), 3.49-3.56 (m, 3H, $\mathrm{CH}(\mathrm{H}) \mathrm{N}_{3, \text { trans }}$ and $\mathrm{CH}(\mathrm{H}) \mathrm{N}_{3, \text { cis }}$ ), 4.31-4.38 (m, $1 \mathrm{H}, \mathrm{CHO}_{\text {trans }}$ ), 4.55 (dddd, $1 \mathrm{H}, J=11.70 \mathrm{~Hz}$, $5.0 \mathrm{~Hz}, 5.0 \mathrm{~Hz}, 2.1 \mathrm{~Hz}, \mathrm{CHO}_{c i s}$ ), 4.62 (dd, $1 \mathrm{H}, J=11.56 \mathrm{~Hz}, 4.40 \mathrm{~Hz}, \mathrm{CHN}_{c i s}$ ), 4.82 (dt, $1 \mathrm{H}, J$ $=5.8 \mathrm{~Hz}, 3.0 \mathrm{~Hz}, \mathrm{CHN}_{\text {trans }}$ ), 5.26 (br s, $1 \mathrm{H}, \mathrm{NH}_{\text {cis }}$ ), 5.58 (br s, $1 \mathrm{H}, \mathrm{NH}_{\text {trans }}$ ), 7.29-7.44 (m, $10 \mathrm{H}$, $\left.2 \mathrm{xC}_{6} \mathrm{H}_{5}\right) .{ }^{13} \mathrm{C} \mathrm{NMR}\left(75 \mathrm{MHz}, \mathrm{CDCl}_{3}\right): \delta=31.7,33.9,52.4,53.6,53.8,55.3,71.9,75.4,125.8$, 126.0, 128.2, 128.9, 129.1, 129.3, 140.1, 141.3, 153.0, 153.2. IR (ATR, $\left.\mathrm{cm}^{-1}\right): v=3239(\mathrm{NH})$, 3123, $2098\left(\mathrm{~N}_{3}\right), 1696(\mathrm{C}=\mathrm{O})$. MS (ES, pos): m/z $233\left(\mathrm{M}+\mathrm{H}^{+}, 100\right)$. Purity $>96 \%$ as determined by reverse phase HPLC analysis (integration at $220 \mathrm{~nm}$ ).

4.2.29. 6-(Azidomethyl)-4-(4-methoxyphenyl)-1,3-oxazinan-2-one (17b). Mixture of cisand trans-isomer in a ratio 82/18. Amorphous grey solid; mp: $158.1-160.1{ }^{\circ} \mathrm{C}$; yield $85 \%$. ${ }^{1} \mathrm{H}$ NMR (300 MHz, $\left.\mathrm{CDCl}_{3}\right): \delta=1.88\left(\mathrm{dt}, 1 \mathrm{H}, J=13.76 \mathrm{~Hz}, 11.7 \mathrm{~Hz}, 5-\mathrm{CH}(\mathrm{H})_{c i s}\right), 1.89-1.96$ (m, 1H, 5-CH(H) $)_{\text {trans }}$ ), 2.11-2.19 (m, 1H, 5- $\mathrm{CH}(H)_{\text {cis }}$ ), 2.30 (ddd, $1 \mathrm{H}, J=13.9 \mathrm{~Hz}, 10.3 \mathrm{~Hz}, 5.9 \mathrm{~Hz}$, 5- $\mathrm{CH}(H)_{\text {trans }}$ ), 3.43 (dd, $1 \mathrm{H}, J=12.93 \mathrm{~Hz}, 5.23 \mathrm{~Hz}, \mathrm{CH}(\mathrm{H}) \mathrm{N}_{3, \text { trans }}$ ), 3.47-3.53 (m, $1 \mathrm{H}$, $\mathrm{CH}(H) \mathrm{N}_{3, \text { trans }}$ ), 3.53 (dd, $\left.1 \mathrm{H}, J=13.21 \mathrm{~Hz}, 4.95 \mathrm{~Hz}, \mathrm{CH}(\mathrm{H}) \mathrm{N}_{3, c i s}\right), 3.54$ (dd, $1 \mathrm{H}, J=13.21 \mathrm{~Hz}$, 
$4.95 \mathrm{~Hz}, \mathrm{CH}(H) \mathrm{N}_{3, \text { cis }}$ ), 3.81 (s, $3 \mathrm{H}, \mathrm{CH}_{3} \mathrm{O}_{\text {trans }}$ ), 3.82 (s, 3H, $\mathrm{CH}_{3} \mathrm{O}_{\text {cis }}$ ), 4.33 (dddd, $1 \mathrm{H}, J=$ $10.25 \mathrm{~Hz}, 5.0 \mathrm{~Hz}, 5.0 \mathrm{~Hz}, 2.7 \mathrm{~Hz}, \mathrm{CHO}_{\text {trans }}$ ), 4.48-4.56 (m, 1H, $\mathrm{CHO}_{\text {cis }}$ ), 4.56 (dd, $1 \mathrm{H}, J=$ $11.42 \mathrm{~Hz}, 4.5 \mathrm{~Hz}, \mathrm{CHN}_{c i s}$ ), 4.76 (dt, 1H, J = 5.5 Hz, 3.2 Hz, CHN trans $_{\text {) }}, 5.47$ (br s, $1 \mathrm{H}, \mathrm{NH}_{c i s}$ ), 5.94 (br s, $1 \mathrm{H}, \mathrm{NH}_{\text {trans }}$ ), 6.89-6.94 (m, 4H, Ar-H), 7.20-7.27 (m, 4H, Ar-H). ${ }^{13} \mathrm{C}$ NMR (75 $\left.\mathrm{MHz}, \mathrm{CDCl}_{3}\right): \delta=31.8,33.9,51.8,53.6,53.8,54.7,55.4,71.9,75.4,114.3,114.5,126.9$, 127.4, 132.0, 133.2, 153.0, 153.3, 159.4, 159.9. IR (ATR, $\left.\mathrm{cm}^{-1}\right): v=3325(\mathrm{NH}), 2138\left(\mathrm{~N}_{3}\right)$, $2110\left(\mathrm{~N}_{3}\right), 1711(\mathrm{C}=\mathrm{O})$. MS (ES, pos): m/z $263\left(\mathrm{M}+\mathrm{H}^{+}, 100\right)$. Purity $>95 \%$ as determined by reverse phase HPLC analysis (integration at $220 \mathrm{~nm}$ ).

\subsubsection{General procedure for the synthesis of 4-amino-4-aryl-1-azidobutan-2-ols 18 . To} a solution of 6-(azidomethyl)-1,3-oxazinan-2-one 17 (1 mmol) in $\mathrm{MeOH}(10 \mathrm{~mL})$ was added $2 \mathrm{M} \mathrm{NaOMe}$ in $\mathrm{MeOH}$ (2 mL, $4 \mathrm{mmol}$ ). The solution was stirred under reflux for $48 \mathrm{~h}$, evaporated under reduced pressure, diluted with $\mathrm{CH}_{2} \mathrm{Cl}_{2}(50 \mathrm{~mL})$ and $\mathrm{H}_{2} \mathrm{O}(50 \mathrm{~mL})$. The aqueous layer was extracted with $\mathrm{CH}_{2} \mathrm{Cl}_{2}(2 \times 50 \mathrm{~mL})$. The combined organic layers were dried $\left(\mathrm{MgSO}_{4}\right)$, filtered and evaporated under reduced pressure. Purification by column chromatography with EtOAc afforded the pure aminoalcohol 18 in 81-90\% yield.

4.2.31. 4-Amino-1-azido-4-phenylbutan-2-ol 18a. Mixture of syn- and anti-isomer in a ratio 78/22. Amorphous yellowish solid; mp: 52.5-55.2 ${ }^{\circ} \mathrm{C}$; yield $90 \% .{ }^{1} \mathrm{H}$ NMR $(300 \mathrm{MHz}$, $\left.\mathrm{CDCl}_{3}\right): \delta=1.73-1.84\left(\mathrm{~m}, 3 \mathrm{H}, 3-\mathrm{CH}(H)_{\text {syn }}\right.$ and 3-CH(H) ${ }_{\text {anti }}$ ), 1.93 (ddd, $1 \mathrm{H}, J=14.31 \mathrm{~Hz}$,

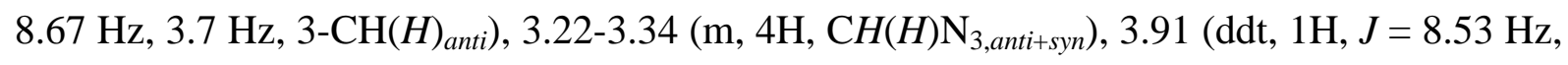
$5.4 \mathrm{~Hz}, 3.03 \mathrm{~Hz}, \mathrm{CHO}_{a n t i}$ ), 4.02-4.07 (m, 1H, $\mathrm{CHN}_{\text {syn }}$ ), 4.10-4.17 (m, 1H, $\mathrm{CHO}_{\text {syn }}$ ), 4.46 (dd, $\left.1 \mathrm{H}, J=6.60 \mathrm{~Hz}, 3.58 \mathrm{~Hz}, \mathrm{CHN}_{a n t i}\right), 7.23-7.40$ (m, 10H, Ar- $\left.\mathrm{H}_{\text {anti+syn }}\right) .{ }^{13} \mathrm{C}$ NMR (75 MHz, $\left.\mathrm{CDCl}_{3}\right): \delta=40.3,40.6,53.7,56.7,56.9$ (2C), 68.7, 71.1, 125.4, 125.9, 127.51, 127.54, 128.9, 129.1, 144.2, 146.4. IR (ATR, $\left.\mathrm{cm}^{-1}\right): v=3356(\mathrm{OH}), 3288(\mathrm{NH}), 2095\left(\mathrm{~N}_{3}\right)$. MS (ES, pos): $\mathrm{m} / \mathrm{z} 207\left(\mathrm{M}+\mathrm{H}^{+}, 100\right)$. Purity $>92 \%$ as determined by reverse phase HPLC analysis (integration at $220 \mathrm{~nm}$ ).

4.2.32. syn-4-Amino-1-azido-4-(4-methoxyphenyl)butan-2-ol 18b. Isolated in a syn/anti ratio >90/10. Amorphous yellowish solid; mp: 63.1-65.8 ${ }^{\circ} \mathrm{C}$; yield 81\%. ${ }^{1} \mathrm{H}$ NMR $(300 \mathrm{MHz}$, $\left.\mathrm{CDCl}_{3}\right): \delta=1.72-1.85(\mathrm{~m}, 2 \mathrm{H}, 3-\mathrm{CH}(H)), 3.25$ (dd, $\left.1 \mathrm{H}, J=12.38 \mathrm{~Hz}, 5.78 \mathrm{~Hz}, \mathrm{CH}(\mathrm{H}) \mathrm{N}_{3}\right)$, 3.30 (dd, $1 \mathrm{H}, J=12.66 \mathrm{~Hz}, 5.0 \mathrm{~Hz}, \mathrm{CH}(H) \mathrm{N}_{3}$ ), 3.81 (s, 3H, $\mathrm{CH}_{3} \mathrm{O}$ ), 3.98-4.03 (m, 1H, CHN), 4.07-4.15 (m, 1H, CHO), 4.46 (dd, 1H, J=6.60 Hz, 3.58 Hz, CHN ${ }_{\text {anti }}$ ), 6.87-6.90 (m, 2H, Ar- 
H), 7.15-7.19 (m, 2H, Ar-H). ${ }^{13} \mathrm{C}$ NMR (75 $\left.\mathrm{MHz} \mathrm{CDCl}_{3}\right): \delta=40.5$, 55.3, 56.1, 56.8, 71.9, 114.2, 126.4, 138.6, 158.8. IR (ATR, $\left.\mathrm{cm}^{-1}\right): v=3366(\mathrm{OH}), 3293(\mathrm{NH}), 2097\left(\mathrm{~N}_{3}\right)$. MS (ES, pos): $\mathrm{m} / \mathrm{z}$ no $\mathrm{M}+\mathrm{H}^{+}, 192$ (100). Purity $>87 \%$ as determined by reverse phase HPLC analysis (integration at $220 \mathrm{~nm}$ ).

\subsubsection{General procedure for the synthesis of 4-amino-4-aryl-1-(phenylselanyl)butan-2-}

ols 19. To a solution of 6-(phenylselanylmethyl)-1,3-oxazinan-2-one 10 (1 mmol) in $\mathrm{MeOH}$ $(10 \mathrm{~mL})$ was added $2 \mathrm{M} \mathrm{NaOMe}$ in $\mathrm{MeOH}$ (2 mL, $4 \mathrm{mmol})$. The solution was stirred under reflux for 36-40 h, evaporated under reduced pressure, diluted with $\mathrm{CH}_{2} \mathrm{Cl}_{2}(50 \mathrm{~mL})$ and $\mathrm{H}_{2} \mathrm{O}$ (50 mL). The aqueous layer was extracted with $\mathrm{CH}_{2} \mathrm{Cl}_{2}(2 \times 50 \mathrm{~mL})$. The combined organic layers were dried $\left(\mathrm{MgSO}_{4}\right)$, filtered and evaporated under reduced pressure. Purification by column chromatography with EtOAc afforded the pure aminoalcohol 19 in 80-84\% yield.

4.2.34. 4-Amino-4-phenyl-1-(phenylselanyl)butan-2-ol 19a. Mixture of syn- and antiisomer in a ratio 2/3. Amorphous white solid; mp: 95.7-97.7 ${ }^{\circ} \mathrm{C}$; yield 84\%. ${ }^{1} \mathrm{H}$ NMR (300 $\mathrm{MHz}, \mathrm{CDCl}_{3}$ ): $\delta=1.75$ (dt, $\left.1 \mathrm{H}, J=14.03 \mathrm{~Hz}, 10.46 \mathrm{~Hz}, 3-\mathrm{CH}(\mathrm{H})_{a n t i}\right), 1.90-2.02$ (m, 3H, 3$\mathrm{CH}(H)_{\text {syn }}$ and 3-CH(H) anti), 2.82-3.33 (m, 8H, $\mathrm{CH}(H) \mathrm{Se}_{\text {anti+syn }}$ and $\left.\mathrm{NH}_{2, \text { anti+syn }}\right), 3.87-3.95(\mathrm{~m}$, $1 \mathrm{H}, \mathrm{CHO}_{\text {syn }}$ ), 4.01 (dd, 1H, J = $10.73 \mathrm{~Hz}, 2.75 \mathrm{~Hz}, \mathrm{CHN}_{\text {anti }}$ ), 4.04-4.12 (m, 1H, $\mathrm{CHO}_{\text {anti }}$ ), 4.33 (t, $\left.1 \mathrm{H}, J=5.6 \mathrm{~Hz}, \mathrm{CHN}_{\text {syn }}\right), 7.19-7.53\left(\mathrm{~m}, 20 \mathrm{H}, \mathrm{Ar}-\mathrm{H}_{\text {anti+syn }}\right) .{ }^{13} \mathrm{C} \mathrm{NMR}\left(75 \mathrm{MHz}, \mathrm{CDCl}_{3}\right): \delta=$ 35.7, 35.9, 43.0, 43.3, 53.4, 56.7, 68.4, 71.9, 125.6, 126.0, 126.9, 127.1, 127.3, 127.4, 128.8, 129.0, 129.2, 130.0, 130.6, 132.5, 133.0, 145.1, 146.6. IR (ATR, $\mathrm{cm}^{-1}$ ): $v=3342$ (OH), 3273 (NH). MS (ES, $\mathrm{M}+\mathrm{H}^{+}$, pos): $\mathrm{m} / \mathrm{z}$ 322/320 $\left(\mathrm{M}+\mathrm{H}^{+}, 100\right)$. Anal. Calcd. for $\mathrm{C}_{16} \mathrm{H}_{19} \mathrm{NOSe}$ C, 60.00; H, 5.98; N, 4.37. Found: C, 60.36; H, 5.69; N, 4.37.

4.2.35. 4-Amino-4-(methoxyphenyl)-1-(phenylselanyl)butan-2-ol 19b. Mixture of syn- and anti-isomer in a ratio 49/51. Amorphous white solid; mp: $101.3-103.3{ }^{\circ} \mathrm{C}$; yield $80 \%$. ${ }^{1} \mathrm{H}$ NMR (300 MHz, $\mathrm{CDCl}_{3}$ ): $\delta=1.73\left(\mathrm{dt}, 1 \mathrm{H}, J=14.03 \mathrm{~Hz}, 10.46 \mathrm{~Hz}, 3-\mathrm{CH}(\mathrm{H})_{\text {anti }}\right.$ ), 1.88-1.99 $\left(\mathrm{m}, 3 \mathrm{H}, 3-\mathrm{CH}(H)_{s y n}\right.$ and 3-CH(H)anti), 2.95-3.11 (m, 4H, CH(H)Se $\left.{ }_{a n t i+s y n}\right), 3.80$ (s, 3H, $\mathrm{CH}_{3} \mathrm{O}_{\text {syn }}$ ), 3.81 (s, 3H, $\mathrm{CH}_{3} \mathrm{O}_{\text {anti) }}$ ), 3.86-3.947 (m, $1 \mathrm{H}, \mathrm{CHO}_{\text {syn }}$ ), 3.97 (dd, $1 \mathrm{H}, J=10.73 \mathrm{~Hz}$, $\left.3.03 \mathrm{~Hz}, \mathrm{CHN}_{a n t i}\right)$, 4.03-4.11 (m, 1H, $\left.\mathrm{CHO}_{a n t i}\right), 4.30\left(\mathrm{t}, 1 \mathrm{H}, J=5.6 \mathrm{~Hz}, \mathrm{CHN}_{\text {syn }}\right), 6.85-7.53(\mathrm{~m}$, 18H, Ar- $\left.\mathrm{H}_{\text {anti+syn }}\right) .{ }^{13} \mathrm{C} \mathrm{NMR}\left(75 \mathrm{MHz}, \mathrm{CDCl}_{3}\right): \delta=35.7,35.9,43.0$, 43.4, 52.9, 55.4, 56.1, 68.4, 71.8, 114.1, 114.2, 126.6, 126.9, 127.0, 127.1, 129.2, 130.0, 130.6, 132.5, 133.0, 137.1, 139.0, 158.8. IR (ATR, $\left.\mathrm{cm}^{-1}\right): v=3338(\mathrm{OH}), 3259(\mathrm{NH}) . \mathrm{MS}\left(\mathrm{ES}, \mathrm{M}+\mathrm{H}^{+}\right.$, pos): $\mathrm{m} / \mathrm{z}$ 333/335 
(100). Anal. Calcd. for $\mathrm{C}_{17} \mathrm{H}_{21} \mathrm{NO}_{2}$ Se: C, 58.29; H, 6.04; N, 4.00. Found: C, 57.97; H, 6.36; N, 4.15 .

4.3. Crystal Data. Compound cis-10b was crystallized (colorless blocks) by slow evaporation from a diethyl ether solution at room temperature. $\mathrm{C}_{12} \mathrm{H}_{14} \mathrm{BrNO}_{3}, \mathrm{M}_{\mathrm{r}}=300.15$, monoclinic, space group $\mathrm{P} 2{ }_{1} / \mathrm{c}, \mathrm{a}=14.6488(9) \AA, \mathrm{b}=6.2920(4) \AA, \mathrm{c}=15.2272(7) \AA, \mathrm{U}=1275.36(13)$ $\AA^{3}, Z=4, T=173(2) K, 9756$ reflections measured, 2290 unique $\left(R_{i n t}=0.057\right)$. The final $\mathrm{wR}\left(\mathrm{F}^{2}\right)$ was 0.088 (all data). Crystallographic data for the structural analysis of compound cis10b have been deposited at the Cambridge Crystallographic Data Centre. The CCDC 767171 has been assigned to the compound cis-10b. Copies of the information may be obtained free of charge from The Director, CCDC, 12 Union Road, Cambridge CB2 1EZ, UK (fax: +441223-336033; e-mail: deposit@ccdc.cam.ac.uk or www: http://www.ccdc.cam.ac.uk).

\section{Acknowledgements}

The authors are indebted to the Research Foundation - Flanders (FWO - Flanders), Ghent University (BOF) and Mersin University (project grant BAP-SBE AKB (YN) 2009-3 DR) for financial support.

\section{References and notes}

1 Zanatta, N.; Borchhardt, D. M.; Alves, S. H.; Coelho, H. S.; Squizani, A. M. C.; Marchi, T. M.; Bonacorso, H. G.; Martins, M. A. P. Bioorg. Med. Chem. 2006, 14, 3174.

2 (a) Wang, G. Anti-Infect. Agents Med. Chem. 2008, 7, 32. (b) Wang, G.; Ella-Menye, J.-R.; Sharma, V. Bioorg. Med. Chem. Lett. 2006, 16, 2177.

3 Ullrich, T.; Baumann, K.; Welzenbach, K.; Schmutz, S.; Camenisch, G.; Meingassner, J. G.;

Weitz-Schmidt, G. Bioorg. Med. Chem. Lett. 2004, 14, 2483.

4 Pedersen, O. S.; Pedersen, E. B. Antiviral. Chem. Chemother. 1999, 10, 285.

5 Jin, F.; Confalone, P. N. PCT Int. Appl. WO0000481, 2000, 119 pp; Chem. Abstr. 2000, 132, 78560 .

6 (a) Wang, Y.-F.; Izawa, T.; Kobayashi, S.; Ohno, M. J. Am. Chem. Soc. 1982, 104, 6465. (b) Kuznetsov, N. Y.; Khrustalev, V. N.; Godovikov, I. A.; Bubnov, Y. N. Eur. J. Org. Chem. 2007, 2015. (c) Woodward, R. B.; Logusch, E.; Nambiar, K. P.; Sakan, K.; Ward, D. E.; AuYeung, B.-W.; Balaram, P.; Browne, L. J.; Card, P. J.; Chen, C. H.; Chênevert, R. B.; Fliri, A.; Frobel, K.; Gais, H.-J.; Garratt, D. G.; Hayakawa, K.; Heggie, W.; Hesson, D. P.; Hoppe, D.; 
Hoppe, I.; Hyatt, J. A.; Ikeda, D.; Jacobi, P. A.; Kim, K. S.; Kobuke, Y.; Kojima, K.;

Krowicki, K.; Lee, V. J.; Leutert, T.; Malchenko, S.; Martens, J.; Matthews, R. S.; Ong, B. S.; Press, J. B.; Rajan Babu, T. V.; Rousseau, G.; Sauter, H. M.; Suzuki, M.; Tatsuta, K.; Tolbert, L. M.; Truesdale, E. A.; Uchida, I.; Ueda, Y.; Uyehara, T.; Vasella, A. T.; Vladuchick, W. C.; Wade, P. A.; Williams, R. M.; Wong H. N.-C. J. Am. Chem. Soc. 1981, 103, 3213. (d) Davies, S. G.; Haggitt, J. R.; Ichihara, O.; Kelly, R. J.; Leech, M. A.; Price Mortimer, A. J.; Roberts, P. M.; Smith, A. D. Org. Biomol. Chem. 2004, 2, 2630. (e) Takahata, H.; Ouchi, H.; Ichinose, M.; Nemoto, H. Org. Lett. 2002, 4, 3459. (f) Jung, J.-W.; Shin, D.-Y.; Seo, S.-Y.; Kim, S.-H.; Paek, S.-M.; Jung, J.-K.; Suh, Y.-G. Tetrahedron Lett. 2005, 46, 573. (g) Puigbó, G.; Diaba, F.; Bonjoch, J. Tetrahedron 2003, 59, 2657. (h) Davies, S. G.; Ichihara, O. Tetrahedron Lett. 1999, 40, 9313.

7 (a) Davies, S. G.; Garner, A. C.; Roberts, P. M.; Smith, A. D.; Sweet, M. J.; Thomson, J. E. Org. Biomol. Chem. 2006, 4, 2753. (b) Vanucci, C.; Brusson, X.; Verdel, V.; Zana, F.;

Dhimane, H.; Lhommet, G. Tetrahedron Lett. 1995, 36, 2971. (c) Wang, C.; Tunge, J. A. Org. Lett. 2006, 8, 3211.

8 Cassady, J. M.; Chan, K. K.; Floss, H. G.; Leistner, E. Chem. Pharm. Bull. 2004, 52, 1. 9 For some selected examples, see: (a) Lohray, B. B.; Baskaran, S.; Reddy, B. Y.; Rao, K. S. Tetrahedron Lett. 1998, 39, 6555. (b) Ella-Menye, J.-R.; Sharma, V.; Wang, G. J. Org. Chem. 2005, 70, 463. (c) Ella-Menye, J.-R.; Wang, G. Tetrahedron 2007, 63, 10034. (d) Schunk, S.; Enders, D. Org. Lett. 2001, 3, 3177. (e) Murakami, M.; Suginome, M.; Fujimoto, K.; Nakamura, H.; Andersson, P. G.; Ito, Y. J. Am. Chem. Soc. 1993, 115, 6487. (f) Hioki, H.; Izawa, T.; Yoshizuka, M.; Kunitake, R.; Itô, S. Tetrahedron Lett. 1995, 36, 2289. 10 (a) González-Rosende, M. E.; Jordá-Gregori, J. M.; Sepúlveda-Arques, J.; Orena, M. Tetrahedron: Asymm. 2004, 15, 419. (b) Bongini, A.; Cardillo, G.; Orena, M. Chem. Lett. 1988, 87.

11 Lait, S. M.; Rankic, D. A.; Keay, B. A. Chem. Rev. 2007, 107, 767.

12 Grajewska, A.; Rozwadowska, M. D. Tetrahedron: Asymm. 2007, 18, 803.

13 Iida, H.; Yamazaki, N.; Kibayashi, C. J. Org. Chem. 1987, 52, 1956.

14 (a) Fuji, K.; Yamada, T.; Fujita, E.; Murata, H. Chem. Pharm. Bull. 1978, 26, 2515. (b) Michael, J. P. Nat. Prod. Rep. 2003, 20, 458. (c) Narasaka, K.; Ukaji, Y.; Yamazaki, S. Bull. Chem. Soc. Jpn. 1986, 59, 525.

15 (a) Malone, M. H.; Rother, A. J. Etnopharm. 1994, 42, 135. (b) Rumalla, C. S.; Jadhav, A. N.; Smillie, T.; Fronczek, F. R.; Khan, I. A. Phytochemistry 2008, 69, 1756. 
16 Weis, R.; Faist, J.; di Vora, U.; Schweiger, K.; Brandner, B.; Kungl, A. J.; Seebacher, W. Eur. J. Med. Chem. 2008, 43, 872.

17 Harris, P. E.; Ferrara, C.; Barba, P.; Polito, T.; Freeby, M.; Maffei, A. J. Mol. Med. 2008, $86,5$.

18 (a) Siddiqui, S. A.; Srinivasan, K. V. Tetrahedron: Asymm. 2007, 18, 2099. (b) Pryor, J. L.; Althof, S. E.; Steidle, C.; Rosen, R. C.; Hellstrom, W. J. G.; Shabsigh, R.; Miloslavsky, M.; Kell, S. Lancet 2006, 368, 929.

19 For selected examples, see: (a) de Figueiredo, R. M.; Fröhlich, R.; Christmann, M. J. Org. Chem. 2006, 71, 4147. (b) Enders, D.; Gries, J. Synthesis 2005, 3508. (c) Barluenga, J.; Tomás, M.; Ballesteros, A.; Kong, J.-S. Tetrahedron 1996, 52, 3095. (d) Barluenga, J.; Fernández-Marí, F.; Viado, A. L.; Aguilar, E.; Olano, B. J. Org. Chem. 1996, 61, 5659. (e) Mangelinckx, S.; Van Speybroeck, V.; Vansteenkiste, P.; Waroquier, M.; De Kimpe, N. J. Org. Chem. 2008, 73, 5481. (f) Aelterman, W.; De Kimpe, N.; Declercq, J.-P. J. Org. Chem. 1998, 63, 6. (g) Ghorai, M. K.; Kumar, A.; Halder, S. Tetrahedron 2007, 63, 4779. (h) Ghorai, M. K.; Das, K.; Kumar, A. Tetrahedron Lett. 2007, 48, 2471. (i) Ghorai, M. K.; Das, K.; Kumar, A.; Das, A. Tetrahedron Lett. 2006, 47, 5393. (j) Morimoto, H.; Wiedemann, S. H.; Yamaguchi, A.; Harada, S.; Chen, Z.; Matsunaga, S.; Shibasaki, M. Angew. Chem. Int. Ed. 2006, 45, 3146.

20 Etxebarria, J.; Vicario, J. L.; Badía, D.; Carrillo, L. Tetrahedron 2007, 63, 11421.

21 D’hooghe, M.; Dekeukeleire S.; Mollet, K.; Lategan, C.; Smith, P. J.; Chibale, K.; De Kimpe, N. J. Med. Chem. 2009, 4058.

22 (a) Yamamoto, Y.; Komatsu, T.; Muruyama, K. J. Org. Chem. 1985, 50, 3115. (b) Yamamoto, Y.; Nishii, S.; Muruyama, K.; Komatsu, T.; Ito, W. J. Am. Chem. Soc. 1986, 108, 7778. (c) David, M.; Dhimane, H.; Vanucci-Bacqué, C.; Lhommet, G. J. Org. Chem. 1999, 64, 8402. (d) Jones, A. D.; Knight, D. W.; Hibbs, D. E. J. Chem. Soc., Perkin Trans. 1 2001, 1182. 23 Phukan, P. J. Org. Chem. 2004, 69, 4005.

24 Dondas, H. A.; De Kimpe, N. Tetrahedron Lett. 2005, 46, 4179.

25 Hoang, C. T.; Alezra, V.; Guillot, R.; Kouklovsky, C. Org. Lett. 2007, 9, 2521.

26 Xie, Y.; Raffo, A.; Ichise, M.; Deng, S.; Harris, P. E.; Landry, D. W. Bioorg. Med. Chem. Lett. 2008, 18, 5111.

27 Ashton, M. J.; Hills, S. J.; Newton, C. G.; Taylor, J. B.; Tondu, S. C. D. Heterocycles 1989, 28, 1015.

28 Davis, F. A.; Fang, T.; Chao, B.; Burns, D. M. Synthesis 2000, 2106. 
29 Davis, F. A.; Chao, B.; Fang, T.; Szewczyk, J. M. Org. Lett. 2000, 2, 1041.

30 Vasse, J.-L.; Levacher, V.; Bourguignon, J.; Dupas, G. Tetrahedron 2003, 59, 4911.

31 Leflemme, N.; Dallemagne, P.; Rault, S. Tetrahedron Lett. 2001, 42, 8997.

32 Kawęcki, R. Tetrahedron 2001, 57, 8385.

33 Oda, R.; Takashima, S.; Okano, M. Bull. Chem. Soc. Jpn. 1962, 35, 1843.

34 Chanet-Ray, J.; Charmier-Januario, M. O.; Vessiere, R.; Zuccarelli, M. J. Heterocycl. Chem. 1994, 31, 1667.

35 Itoh, J.; Fuchibe, K.; Akiyama, T. Angew. Chem. Int. Ed. 2006, 45, 4796.

36 (a) Martin, J. C.; Hoyle, V. A., Jr.; Brannock, K. C. Tetrahedron Lett. 1965, 3589. (b)

Martin, J. C.; Brannock, K. C.; Burpitt, R. D.; Gott, P. G.; Hoyle, V. A., Jr. J. Org. Chem. 1971, 36, 2211.

37 Matsuura, F.; Hamada, Y.; Shiori, T. Tetrahedron Lett. 1994, 35, 733.

38 (a) Bell, E. A.; Tirimanna, A. S. L. Biochem. J. 1964, 91, 356. (b) Pandey, S. K.; Pandey, M.; Kumar, P. Tetrahedron Lett. 2008, 49, 3297.

39 (a) von Nussbaum, F.; Brands, M.; Hinzen, B.; Weigand, S.; Häbich, D. Angew. Chem. Int. Ed. 2006, 45, 5072. (b) Waldmann, H.; He, Y.-P.; Tan, H.; Arve, L. Arndt, H.-D. Chem. Commun. 2008, 5562.

40 Kondo, S.; Meguriya, N.; Mogi, H.; Aota, T.; Miura, K.; Fujii, T.; Hayashi, I.; Makino, K.; Yamamoto, M. Nakajima, N. J. Antibiot. 1980, 33, 533.

41 (a) Pruess, D. L.; Kellet, M. J. Antibiot. 1983, 36, 208; (b) Muller, J. C.; Toome, V.; Pruess, D. L.; Blount, J. F.; Weigele, M. J. Antibiot. 1983, 36, 217.

42 Freudendahl, D. M.; Shahzad, S. A.; Wirth, T. Eur. J. Org. Chem. 2009, 1649.

43 Geach, N. J.; Gilmour, J.; Hatton, L. R.; Smith, P. H. G. Eur. Pat. Appl. 278742 1988;

Chem. Abstr. 1989, 110, 38898. 